\title{
Neutron physics of the Re/Os clock. III. Resonance analyses and stellar $(n, \gamma)$ cross sections of ${ }^{186,187,188}$ Os
}

K. Fujii,,$^{1,}$ M. Mosconi, ${ }^{2}$ A. Mengoni, ${ }^{3,18}$ C. Domingo-Pardo, ${ }^{2,4}$ F. Käppeler, ${ }^{2}$ U. Abbondanno, ${ }^{1}$ G. Aerts, ${ }^{5}$ H. Álvarez-Pol, ${ }^{6}$ F. Alvarez-Velarde,${ }^{7}$ S. Andriamonje,${ }^{5}$ J. Andrzejewski, ${ }^{8}$ P. Assimakopoulos, ${ }^{9}$ L. Audouin, ${ }^{2}$ G. Badurek, ${ }^{10}$ P. Baumann, ${ }^{11}$ F. Bečvář, ${ }^{12}$ F. Belloni, ${ }^{1}$ E. Berthoumieux, ${ }^{5}$ S. Bisterzo, ${ }^{2,13}$ M. Calviani, ${ }^{14}$ F. Calviño, ${ }^{15}$ D. Cano-Ott, ${ }^{7}$ R. Capote,${ }^{3,16}$ A. Carrillo de Albornoz, ${ }^{17}$ P. Cennini, ${ }^{18}$ V. Chepel, ${ }^{19}$ E. Chiaveri, ${ }^{18}$ N. Colonna, ${ }^{20}$ G. Cortes, ${ }^{15}$ A. Couture, ${ }^{21}$ J. Cox,${ }^{21}$ M. Dahlfors, ${ }^{18}$ S. David, ${ }^{22}$ I. Dillmann, ${ }^{2}$ R. Dolfini, ${ }^{23}$ W. Dridi, ${ }^{5}$ I. Duran, ${ }^{6}$ C. Eleftheriadis, ${ }^{24}$ M. Embid-Segura, ${ }^{7}$ L. Ferrant, ${ }^{22}$ A. Ferrari, ${ }^{18}$ R. Ferreira-Marques, ${ }^{19}$ L. Fitzpatrick, ${ }^{18}$ H. Frais-Koelbl, ${ }^{3}$ W. Furman, ${ }^{25}$ R. Gallino, ${ }^{13}$ I. Goncalves, ${ }^{19}$

E. Gonzalez-Romero, ${ }^{7}$ A. Goverdovski, ${ }^{26}$ F. Gramegna, ${ }^{14}$ E. Griesmayer, ${ }^{3}$ C. Guerrero, ${ }^{7}$ F. Gunsing, ${ }^{5}$ B. Haas, ${ }^{27}$ R. Haight, ${ }^{28}$ M. Heil, ${ }^{2}$ A. Herrera-Martinez, ${ }^{18}$ M. Igashira, ${ }^{29}$ S. Isaev, ${ }^{22}$ E. Jericha, ${ }^{10}$ Y. Kadi, ${ }^{18}$ D. Karamanis, ${ }^{9}$ D. Karadimos, ${ }^{9}$ M. Kerveno, ${ }^{11}$ V. Ketlerov, ${ }^{17,25}$ P. Koehler, ${ }^{30}$ V. Konovalov, ${ }^{17,24}$ E. Kossionides, ${ }^{31}$ M. Krtička, ${ }^{12}$ C. Lamboudis, ${ }^{9}$ H. Leeb, ${ }^{10}$ A. Lindote, ${ }^{19}$ I. Lopes, ${ }^{19}$ M. Lozano, ${ }^{16}$ S. Lukic, ${ }^{11}$ J. Marganiec, ${ }^{8}$ L. Marques, ${ }^{17}$ S. Marrone,${ }^{20}$ C. Massimi, ${ }^{32}$ P. Mastinu, ${ }^{14}$ P. M. Milazzo, ${ }^{1}$ C. Moreau, ${ }^{1}$ F. Neves, ${ }^{19}$ H. Oberhummer, ${ }^{10}$ M. Oshima ${ }^{33}$ S. O’Brien, ${ }^{21}$ J. Pancin, ${ }^{5}$

C. Papachristodoulou, ${ }^{9}$ C. Papadopoulos ${ }^{34}$ C. Paradela,${ }^{6}$ N. Patronis, ${ }^{9}$ A. Pavlik, ${ }^{35}$ P. Pavlopoulos,${ }^{36}$ L. Perrot, ${ }^{5}$ R. Plag, ${ }^{2}$ A. Plompen, ${ }^{37}$ A. Plukis, ${ }^{5}$ A. Poch, ${ }^{15}$ J. Praena, ${ }^{16}$ C. Pretel, ${ }^{15}$ J. Quesada, ${ }^{16}$ T. Rauscher, ${ }^{38}$ R. Reifarth, ${ }^{28}$ M. Rosetti, ${ }^{39}$ C. Rubbia, ${ }^{23}$ G. Rudolf, ${ }^{11}$ P. Rullhusen, ${ }^{37}$ J. Salgado, ${ }^{17}$ L. Sarchiapone, ${ }^{18}$ I. Savvidis, ${ }^{24}$ C. Stephan, ${ }^{22}$ G. Tagliente, ${ }^{20}$ J. L. Tain, ${ }^{4}$ L. Tassan-Got, ${ }^{22}$ L. Tavora, ${ }^{17}$ R. Terlizzi, ${ }^{20}$ G. Vannini, ${ }^{32}$ P. Vaz, ${ }^{17}$ A. Ventura, ${ }^{39}$ D. Villamarin, ${ }^{7}$ M. C. Vincente, ${ }^{7}$ V. Vlachoudis, ${ }^{18}$ R. Vlastou, ${ }^{34}$ F. Voss,${ }^{2}$ S. Walter, ${ }^{2}$ H. Wendler, ${ }^{18}$ M. Wiescher, ${ }^{21}$ and K. Wisshak ${ }^{2}$

(The n_TOF Collaboration)

${ }^{1}$ Istituto Nazionale di Fisica Nucleare, I-34149 Trieste, Italy

${ }^{2}$ Karlsruhe Institute of Technology (KIT), Campus Nord, Institut für Kernphysik, D-76021 Karlsruhe, Germany

${ }^{3}$ International Atomic Energy Agency, NAPC-Nuclear Data Section, A-1400 Vienna, Austria

${ }^{4}$ Instituto de Física Corpuscular, CSIC-Universidad de Valencia, E-46980 Paterna, Spain

${ }^{5}$ CEA/Saclay, DSM, F-91191 Gif-sur-Yvette, France

${ }^{6}$ Universidade de Santiago de Compostela, 15782 Spain

${ }^{7}$ Centro de Investigaciones Energeticas Medioambientales y Technologicas, E-28040 Madrid, Spain

${ }^{8}$ University of Lodz, PL-90-142 Lodz, Poland

${ }^{9}$ University of Ioannina, GR-451 10 Ioannina, Greece

${ }^{10}$ Atominstitut der Österreichischen Universitäten, Technische Universität Wien, A-1020 Vienna, Austria

${ }^{11}$ Centre National de la Recherche Scientifique/IN2P3, IReS, F-67037 Strasbourg, France

${ }^{12}$ Charles University, CZ-252 41 Prague, Czech Republic

${ }^{13}$ Dipartimento di Fisica Generale, Università di Torino, I-10149 Torino, Italy

${ }^{14}$ Istituto Nazionale di Fisica Nucleare (INFN), Laboratori Nazionali di Legnaro, I-35020 Legnaro (Padova), Italy

${ }^{15}$ Universitat Politecnica de Catalunya, E-08034 Barcelona, Spain

${ }^{16}$ Universidad de Sevilla, E-41004 Sevilla, Spain

${ }^{17}$ Instituto Tecnológico e Nuclear (ITN), P-2686-953 Sacavém (Lisbon), Portugal

${ }^{18}$ CERN, CH-1211 Geneva, Switzerland

${ }^{19}$ LIP, Coimbra \& Departamento de Fisica da Universidade de Coimbra, P-3004-531 Coimbra, Portugal

${ }^{20}$ Istituto Nazionale di Fisica Nucleare, I-70126 Bari, Italy

${ }^{21}$ University of Notre Dame, Notre Dame, IN 46556, USA

${ }^{22}$ Centre National de la Recherche Scientifique/IN2P3, IPN, F-91406 Orsay, France

${ }^{23}$ Università degli Studi Pavia, I-27100 Pavia, Italy

${ }^{24}$ Aristotle University of Thessaloniki, GR-541 24 Thessaloniki, Greece

${ }^{25}$ Joint Institute for Nuclear Research, Frank Laboratory of Neutron Physics, RUS-141980 Dubna, Russia

${ }^{26}$ Institute of Physics and Power Engineering, Kaluga region, RUS-249020 Obninsk, Russia

${ }^{27}$ Centre National de la Recherche Scientifique/IN2P3, CENBG, F-33175 Gradignan (Bordeaux), France

${ }^{28}$ Los Alamos National Laboratory, Los Alamos, NM 87545, USA

${ }^{29}$ Tokyo Institute of Technology, Tokyo 152-8550, Japan

${ }^{30}$ Oak Ridge National Laboratory, Physics Division, Oak Ridge, TN 37831-6369, USA

${ }^{31}$ NCSR Demokritos, GR-15310 Aghia Paraskevi (Athens), Greece

${ }^{32}$ Dipartimento di Fisica, Università di Bologna, and Sezione INFN di Bologna, I-40126 Bologna, Italy

${ }^{33}$ Japan Atomic Energy Research Institute, Tokai-mura, Ibaraki 319-1184, Japan

${ }^{34}$ National Technical University of Athens, GR-10682 Athens, Greece

${ }^{35}$ Faculty of Physics, University of Vienna, A-1010 Vienna, Austria

${ }^{36}$ Pôle Universitaire Léonard de Vinci, F-92916 Paris La Défense, France

${ }^{37}$ CEC-JRC-IRMM, B-2440 Geel, Belgium 


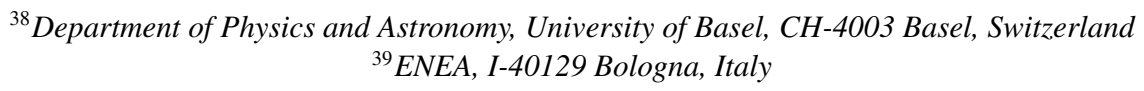

(Received 14 December 2009; published 15 July 2010)

\begin{abstract}
Neutron resonance analyses have been performed for the capture cross sections of ${ }^{186} \mathrm{Os}$, ${ }^{187} \mathrm{Os}$, and ${ }^{188} \mathrm{Os}$ measured at the n_TOF facility at CERN. Resonance parameters have been extracted up to 5, 3, and $8 \mathrm{keV}$, respectively, using the SAMMY code for a full $R$-matrix fit of the capture yields. From these results average resonance parameters were derived by a statistical analysis to provide a comprehensive experimental basis for modeling of the stellar neutron capture rates of these isotopes in terms of the Hauser-Feshbach statistical model. Consistent calculations for the capture and inelastic reaction channels are crucial for the evaluation of stellar enhancement factors to correct the Maxwellian averaged cross sections obtained from experimental data for the effect of thermally populated excited states. These factors have been calculated for the full temperature range of current scenarios of $s$-process nucleosynthesis using the combined information of the experimental data in the region of resolved resonances and in the continuum. The consequences of this analysis for the $s$-process component of the ${ }^{187} \mathrm{Os}$ abundance and the related impact on the evaluation of the time duration of galactic nucleosynthesis via the Re/Os cosmochronometer are discussed.
\end{abstract}

DOI: 10.1103/PhysRevC.82.015804

PACS number(s): 21.10.Ma, 25.40.Lw, 26.20.Kn, 27.70.+q

\section{INTRODUCTION}

The possibility to determine the age of the galaxy via the $\beta$ decay of ${ }^{187} \operatorname{Re}\left(t_{1 / 2}=41.2\right.$ Gyr [1] $)$ has attracted considerable attention because the related nuclear physics aspects are accessible to experimental studies. Apart from its radiogenic component, ${ }^{187}$ Os is synthesized only by the $s$ process during the asymptotic giant branch (AGB) phase of low-mass stars because it is shielded by ${ }^{187} \mathrm{Re}$ against the neutron-rich side of the stability valley, where the $r$ process takes place. The fact that its neighbor isotope ${ }^{186} \mathrm{Os}$ is of pure $s$-process origin provides a direct way for defining the $s$ component of ${ }^{187}$ Os as well.

The $s$-process chain within the reaction network is sketched in Fig. 1. The resulting abundances can be reliably determined with current stellar models [2,3], provided that the neutron capture cross sections of the involved isotopes are accurately known. This means that the radiogenic component of the ${ }^{187}$ Os abundance, $N_{\mathrm{c}}\left({ }^{187} \mathrm{Os}\right)$, in the solar system can be obtained with confidence for a quantitative determination of the mother/daughter ratio required for the assessment of the $\mathrm{Re} / \mathrm{Os}$ cosmochronometer. In this context it is to be noted that ${ }^{187} \mathrm{Re}$ is essentially produced in the $r$ process, which is believed to occur in supernova explosions of massive stars. Since these stars evolve quickly, the clock started early after galaxy formation. This was confirmed by recent observations of very metal poor stars, which exhibit remarkably consistent $r$-process abundance patterns [4-6].

Following first attempts to use the analytic model of Clayton [7] by Luck et al. [8,9] it turned out that the quantitative analysis of the Re/Os clock is complicated for a number of reasons. Apart from the astrophysical question for the production rate of ${ }^{187} \mathrm{Re}$ with time [10-13], other key problems refer to several nuclear physics issues. Takahashi and Yokoi [14] pointed out that the $\beta$-decay rate of ${ }^{187} \mathrm{Re}$ is drastically enhanced at stellar temperatures in excess of about $10^{8} \mathrm{~K}$,

*Kaori.Fujii@ts.infn.it when the increasing degree of ionization opens additional decay channels to unoccupied atomic orbits (bound state decay). The theoretically expected decay rate for fully stripped ${ }^{187} \mathrm{Re}$ could be quantitatively confirmed by a direct half-life measurement at GSI [15], thus providing a sound solution for the crucial problem that part of ${ }^{187} \mathrm{Re}$ was destroyed in later stellar generations. The corresponding correction of the $\mathrm{Re} / \mathrm{Os}$ clock for this astration effect was conservatively reported to contribute an uncertainty of $2-4$ Gyr to the Re/Os age [16,17].

The second main issue is the separation of the radiogenic component of the ${ }^{187}$ Os abundance, which is obtained by subtraction of the $s$-process component from the solar abundance value,

$$
N_{\mathrm{c}}\left({ }^{187} \mathrm{Os}\right)=N_{\odot}\left({ }^{187} \mathrm{Os}\right)-N_{s}\left({ }^{187} \mathrm{Os}\right) .
$$

A possible small contribution of $\leqslant 1 \%$ from the $p$ process can be neglected in this context as discussed in Sec. IV. The $s$ component of ${ }^{187} \mathrm{Os}$ is determined by the local approximation for the $s$-process reaction chain,

$$
\langle\sigma\rangle N_{s}=\text { const. }
$$

where $\langle\sigma\rangle$ is the Maxwellian average $(n, \gamma)$ cross section (MACS) at the stellar site of the $s$ process and $N_{s}$ the $s$ abundance of a particular isotope. This approximation is well satisfied for the W-Re-Os isotopes, because the MACSs are sufficiently large in this mass region to establish reaction flow equilibrium during the $s$ process [3].

The sketch of the reaction flow in Fig. 1 shows that the immediate neighbor isotope ${ }^{186} \mathrm{Os}$ is of pure $s$ process origin because it is shielded from possible $r$-process contributions by its stable isobar ${ }^{186} \mathrm{~W}$. Therefore, the $s$ component of ${ }^{187} \mathrm{Os}$ can be directly obtained via Eq. (2),

$$
N_{s}\left({ }^{187} \mathrm{Os}\right)=N_{s}\left({ }^{186} \mathrm{Os}\right) \frac{\langle\sigma\rangle\left({ }^{186} \mathrm{Os}\right)}{\langle\sigma\rangle\left({ }^{187} \mathrm{Os}\right)} .
$$

As outlined in Papers I [18] and II [19], the stellar MACS values have to be used in this solution for $N_{s}\left({ }^{187} \mathrm{Os}\right)$, which are obtained by correction of the cross sections measured in laboratory experiments with the proper stellar enhancement 


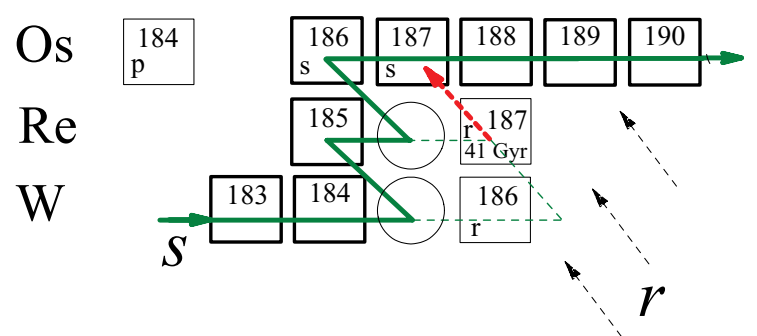

FIG. 1. (Color online) The reaction path of the $s$ process in the W-Re-Os region. The main $s$ process flow is sketched by solid lines, circles correspond to branchings in the $s$ path, and $r$-process contributions from explosive nucleosynthesis are indicated by dashed arrows. The decay of ${ }^{187} \mathrm{Re}$ constitutes a cosmochronometer, which can be analyzed because the radiogenic component of ${ }^{187}$ Os can be determined by subtraction of the $s$-process component. This $s$ component is defined by the abundance of the $s$-only nucleus ${ }^{186} \mathrm{Os}$ via $s$-process systematics.

factors (SEF). These corrections are required because lowlying nuclear states can be thermally populated in the hot and dense stellar site of the $s$ process. The effect of neutron captures on excited states has to be calculated using the HauserFeshbach statistical model (HFSM) [20]. With this approach, reliable cross section calculations can be performed, provided that a consistent set of input parameters has been determined on the basis of experimental information.

In Sec. II, average resonance parameters such as mean level spacings $\left\langle D_{0}\right\rangle$, average radiative widths $\left\langle\Gamma_{\gamma}\right\rangle$, and neutron strength functions $S_{0}$ are derived from comprehensive resonance analyses of the n_TOF data presented in Paper I [18]. These quantities, which are crucial for establishing a reliable parameter set for the HFSM calculations, were complemented by the information on the neutron transmission functions for excited nuclear levels, which could be derived in Paper II from the inelastic-scattering cross section populating the first excited state in ${ }^{187}$ Os [19].

The second part of the article is devoted to the description of the HFSM calculations and to the evaluation of the stellar enhancement factors (Sec. III). At the end, a brief discussion of the consequences of the improved nuclear physics input for the $\mathrm{Re} / \mathrm{Os}$ clock is added in Sec. IV.

\section{RESONANCE ANALYSES}

\section{A. Capture cross sections}

The $(n, \gamma)$ cross sections under analysis were measured at the CERN pulsed neutron facility $n_{-}$TOF [21,22]. Neutrons were generated by spallation reactions induced by a beam of $20 \mathrm{GeV}$ protons with a $6-\mathrm{ns}$ pulse width and $0.4-\mathrm{Hz}$ repetition rate in a massive lead target. In the energy range of the present measurement between $1 \mathrm{eV}$ and $1 \mathrm{MeV}$ the nominal flux at the end of the $185 \mathrm{~m}$ flight path was $2 \times 10^{5}$ neutrons per energy decade per proton bunch. The corresponding resolution in neutron energy was between $3 \times 10^{-4}$ and $4 \times 10^{-3}$.

Capture events were recorded with two $\mathrm{C}_{6} \mathrm{D}_{6}$ scintillation detectors via the prompt $\gamma$-ray cascades. The relative neutron flux was measured upstream of the capture samples with a low
TABLE I. Isotopic composition of the Os samples.

\begin{tabular}{lrrrrrr}
\hline \hline \multirow{2}{*}{ Sample } & \multicolumn{6}{c}{ Isotopic composition (\%) } \\
\cline { 2 - 7 } & ${ }^{186}$ Os & ${ }^{187}$ Os & ${ }^{188}$ Os & ${ }^{189}$ Os & ${ }^{190}$ Os & ${ }^{192}$ Os \\
\hline${ }^{186}$ Os & 78.48 & 0.91 & 4.88 & 4.29 & 5.09 & 5.32 \\
${ }^{187}$ Os & 1.06 & 70.43 & 12.73 & 5.13 & 5.42 & 5.21 \\
${ }^{188}$ Os & 0.11 & 0.12 & 94.99 & 2.55 & 1.27 & 0.97 \\
\hline \hline
\end{tabular}

mass flux monitor consisting of a ${ }^{6} \mathrm{Li}$ layer $200 \mu \mathrm{g} / \mathrm{cm}^{2}$ in thickness. Charged particles from ${ }^{6} \mathrm{Li}(n, \alpha)^{3} \mathrm{H}$ reactions were registered by four silicon detectors outside the neutron beam.

The samples, which were encapsulated in 0.1-mm-thick aluminum cans, consisted of $\approx 2 \mathrm{~g}$ of metal powder with isotopic enrichments between 70 and $95 \%$ (Table I). Additional samples of ${ }^{197} \mathrm{Au},{ }^{\text {nat }} \mathrm{C}$, and ${ }^{\text {nat }} \mathrm{Pb}$ were used for neutron flux normalization and for background measurements.

A detailed description of the measurements and data analysis was presented in Paper I [18].

\section{B. R-matrix fits}

The capture yields in the resolved resonance region were analyzed with the multilevel $R$-matrix code SAMMY [23]. The code was used in the Reich-Moore formalism and included corrections for Doppler and resolution broadening as well as for sample multiple scattering and self-shielding. Resonances due to isotopic impurities (Table I) were properly considered. By means of statistical methods it was verified that all observed resonances can be confidently assumed to be $s$ wave [24].

For resonances with $\Gamma_{n} \gg \Gamma_{\gamma}$, the values for $g \Gamma_{n}$ were adopted from transmission experiments [25], while radiative widths, $\Gamma_{\gamma}$, and resonance energies, $E_{r}$, were determined by the $R$-matrix fit. In these cases, only the SAMMY results for $\Gamma_{\gamma}$ and $E_{r}$ are given with uncertainties in the appendix. Resonance parameters with zero uncertainty were treated as fixed values in the SAMMY fits. In this way, $\Gamma_{\gamma}$ values could be obtained for 52 , 32 , and 61 resonances in ${ }^{186} \mathrm{Os},{ }^{187} \mathrm{Os}$, and ${ }^{188} \mathrm{Os}$, respectively. The average values $\left\langle\Gamma_{\gamma}\right\rangle$ determined from that ensemble were then used in the analysis of resonances with small $\Gamma_{n}$ and for the new resonances, especially at higher neutron energies. In the more difficult cases with $\Gamma_{n} \approx \Gamma_{\gamma}$, the $g \Gamma_{n}$ data of Ref. [25] and $\left\langle\Gamma_{\gamma}\right\rangle$ were basically used as start values in the fits, but the adopted values in the appendix were decided according to the quality of the fits.

Resonance parameters have been extracted up to 5,3 , and $8 \mathrm{keV}$ for ${ }^{186} \mathrm{Os},{ }^{187} \mathrm{Os}$, and ${ }^{188} \mathrm{Os}$, respectively, and new resonances could be resolved for energies higher than 3.4, 1.0, and $4.9 \mathrm{keV}$ in these isotopes. Because there was no information concerning the neutron widths of these new resonances, only the capture kernels $g \Gamma_{\gamma} \Gamma_{n} / \Gamma$ could be determined, where

$$
g_{J}=\frac{(2 J+1)}{(2 s+1)(2 I+1)}
$$

is the statistical weighting factor for target nuclei with spin $I$ and compound states of total angular momentum $J$. The spin of the incident neutron is $s=1 / 2$. Similarly, only capture 

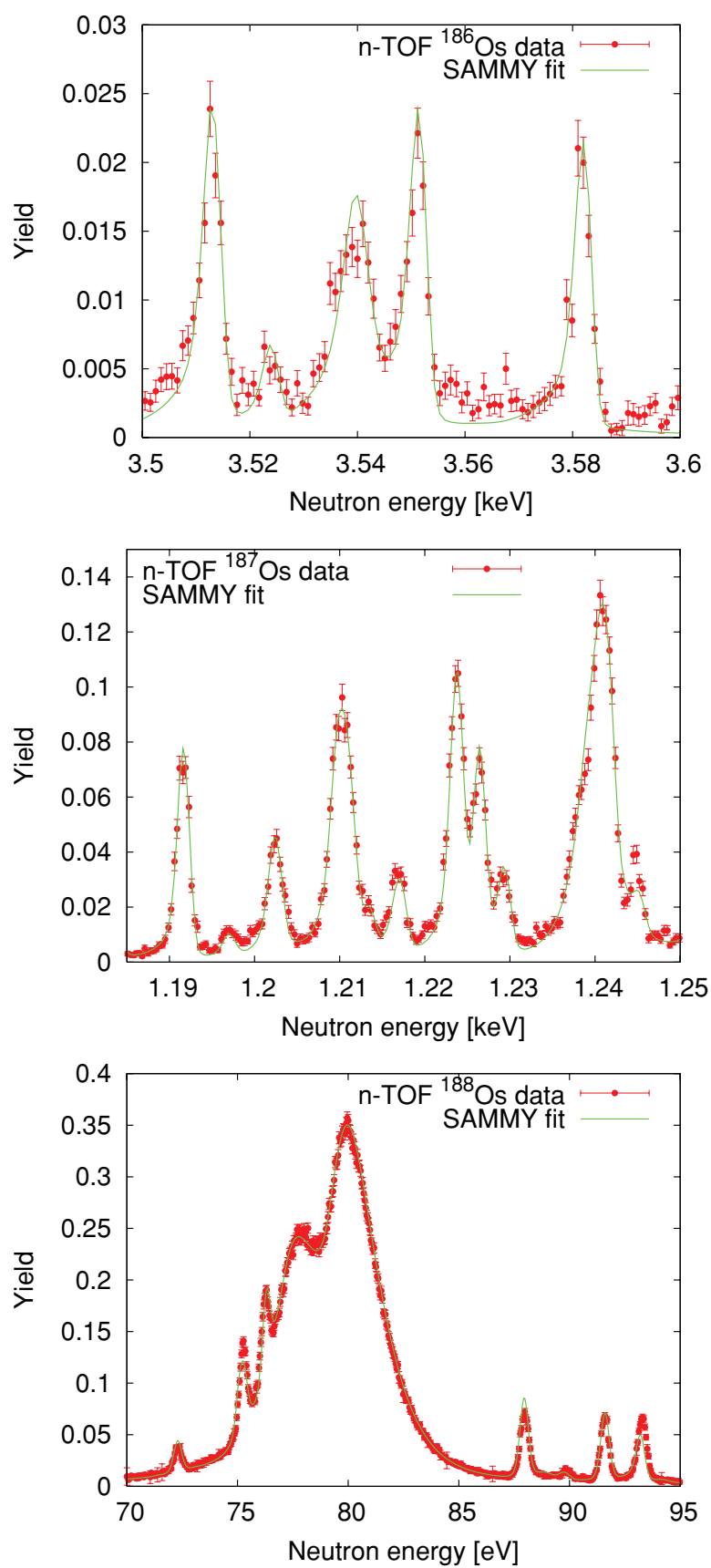

FIG. 2. (Color online) Examples of SAMMY fits of the ${ }^{186,187,188} \mathrm{Os}$ yields, including the resonances of isotopic impurities.

kernels are given for those resonances in the appendix, where suited $g \Gamma_{n}$ values are missing in Ref. [25].

It was found that the first few resonances of ${ }^{186} \mathrm{Os}$ and ${ }^{187} \mathrm{Os}$ could not be fitted because of sample inhomogeneities, which gave rise to strange resonance shapes. Simulations of this effect in transmission data [26] showed that it is determined by the ratio of sample thickness and grain size. Compared to the sample thickness of $0.5 \mathrm{~mm}$ the similar grain size of the Os metal powder of $\approx 0.2$ to $0.5 \mathrm{~mm}$ could in fact explain the problematic resonance shapes of the first strong resonances [27]. The effect of sample inhomogeneities could not be treated with the present version of SAMMY.
The deduced resonance parameters are listed in the appendix. Examples of SAMMY fits are shown in Fig. 2.

\section{Statistical analysis and average quantities}

The present set of resonance parameters was used for a statistical analysis to determine the nuclear properties required for the cross-section calculations described in Sec. III, i.e., average level spacings $\left\langle D_{0}\right\rangle$, average radiative widths $\left\langle\Gamma_{\gamma}\right\rangle$, and neutron strength functions $S_{0}$. The overview in Table II shows the number of analyzed resonances compared to the subset used in the statistical analysis.

The cumulative number of resonances as a function of neutron energy shown in the staircase plots of Fig. 3 provide an efficient way to investigate level populations and missing levels. The average $s$-wave level spacings $\left\langle D_{0}\right\rangle$ are directly related to the inverse slope of these plots and can be obtained by the linear least-squares fits indicated by straight lines. The corresponding entries in Table III have been verified by means of a maximum-likelihood analysis assuming a Wigner distribution for the level spacing. The results of this approach were fully consistent with those from the straight-line fit of the cumulative number of levels.

The average quantities deduced from our data sets of resolved resonance parameters are compared in Table III with the reference values of Mughabghab [25]. The results for $\left\langle D_{0}\right\rangle$ are compatible for the even isotopes within uncertainties but for ${ }^{187}$ Os we find a significant difference. Since the total number of observed resonances in our measurement is almost identical with those in Ref. [25] we believe that this difference is due to the adopted method for extracting $\left\langle D_{0}\right\rangle$.

The distribution of reduced neutron widths $\left(\Gamma_{n}^{0}=\Gamma_{n} / \sqrt{E_{r}}\right)$ from our data sets are compared with the theoretical PorterThomas (PT) distributions in Fig. 4. The good agreement suggests that only a few weak resonances might have been missed in ${ }^{187} \mathrm{Os}$. This has been confirmed by means of the missing level estimator [28] to obtain the average reduced neutron widths.

Assuming a PT distribution for the reduced neutron widths and a Wigner distribution for the level spacing the $s$-wave neutron strength function is

$$
S_{0}=\frac{\left\langle g \Gamma_{n}^{0}\right\rangle}{\left\langle D_{0}\right\rangle}\left(1 \pm \sqrt{\frac{2.27}{N_{0}}}\right),
$$

where $N_{0}$ denotes the number of resonances. The present neutron strength functions agree within the

TABLE II. Overview of the statistical analysis. ${ }^{a}$

\begin{tabular}{llll}
\hline \hline & \multirow{2}{*}{$\begin{array}{c}\text { Number of } \\
\text { analyzed resonances }\end{array}$} & \multicolumn{2}{c}{ Statistical analysis } \\
\cline { 3 - 4 } & $186(5.0)$ & $126(3.4)$ & $122(3.4)$ \\
\hline${ }^{186} \mathrm{Os}$ & $480(3.0)$ & $179(1.0)$ & $327(2.0)$ \\
${ }^{187} \mathrm{Os}$ & $199(8.0)$ & $125(5.0)$ & $125(5.0)$ \\
${ }^{188} \mathrm{Os}$ &
\end{tabular}

${ }^{\mathrm{a}}$ Maximum neutron energies (keV) are given in parentheses. 

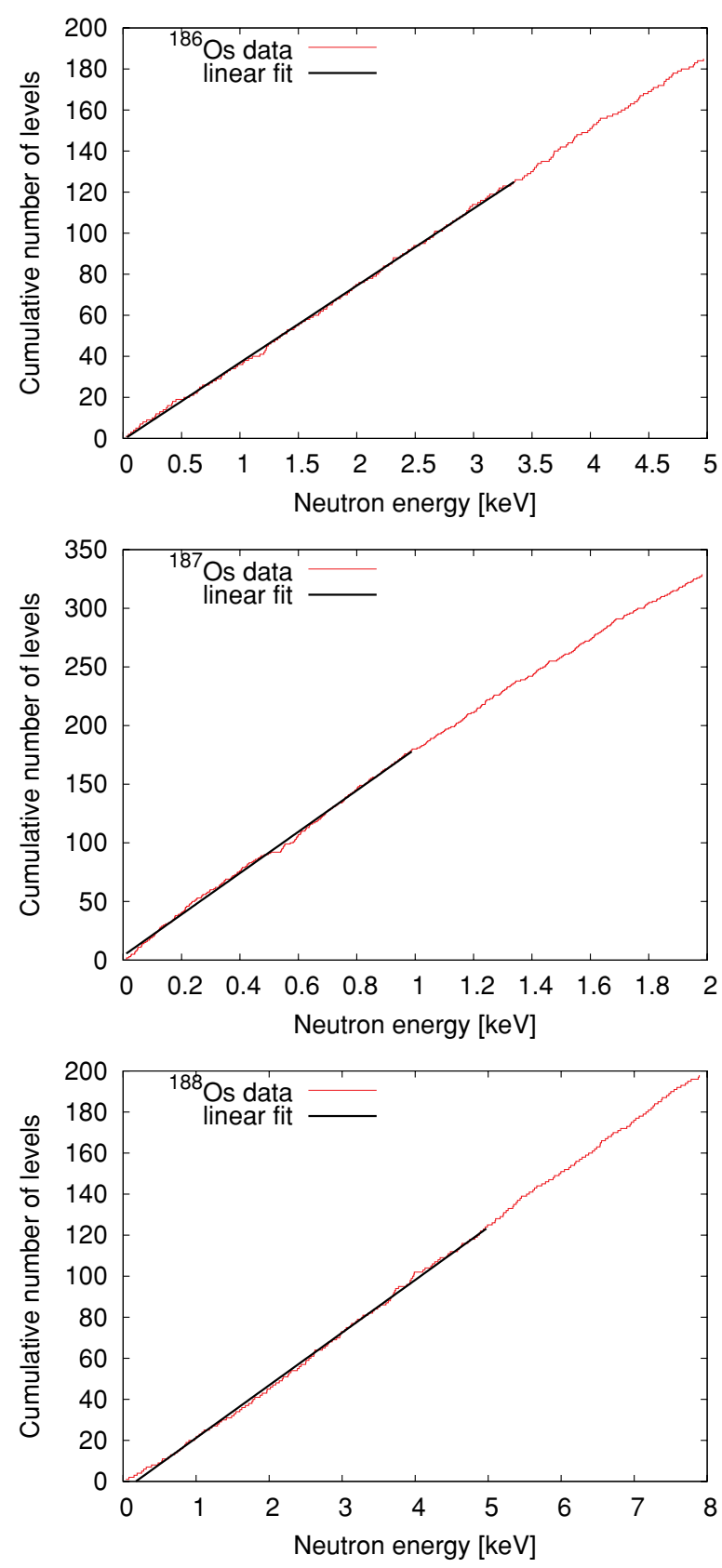

FIG. 3. (Color online) Stair case plots of the cumulative numbers of resonances in the investigated Os isotopes.

(rather large) uncertainties with those of Ref. [25] (Table III).

The average radiative widths could be determined with improved accuracy as described in subsection II B. The present results are consistently smaller than the values in Ref. [25]. This holds in particular for ${ }^{188}$ Os.

\section{CROSS SECTIONS AT STELLAR TEMPERATURES}

A reliable theoretical description of $(n, \gamma)$ cross sections is crucial for evaluating the effect of excited states, which are thermally populated at the high temperatures at the stellar site of the $s$ process. The average resonance parameters obtained as
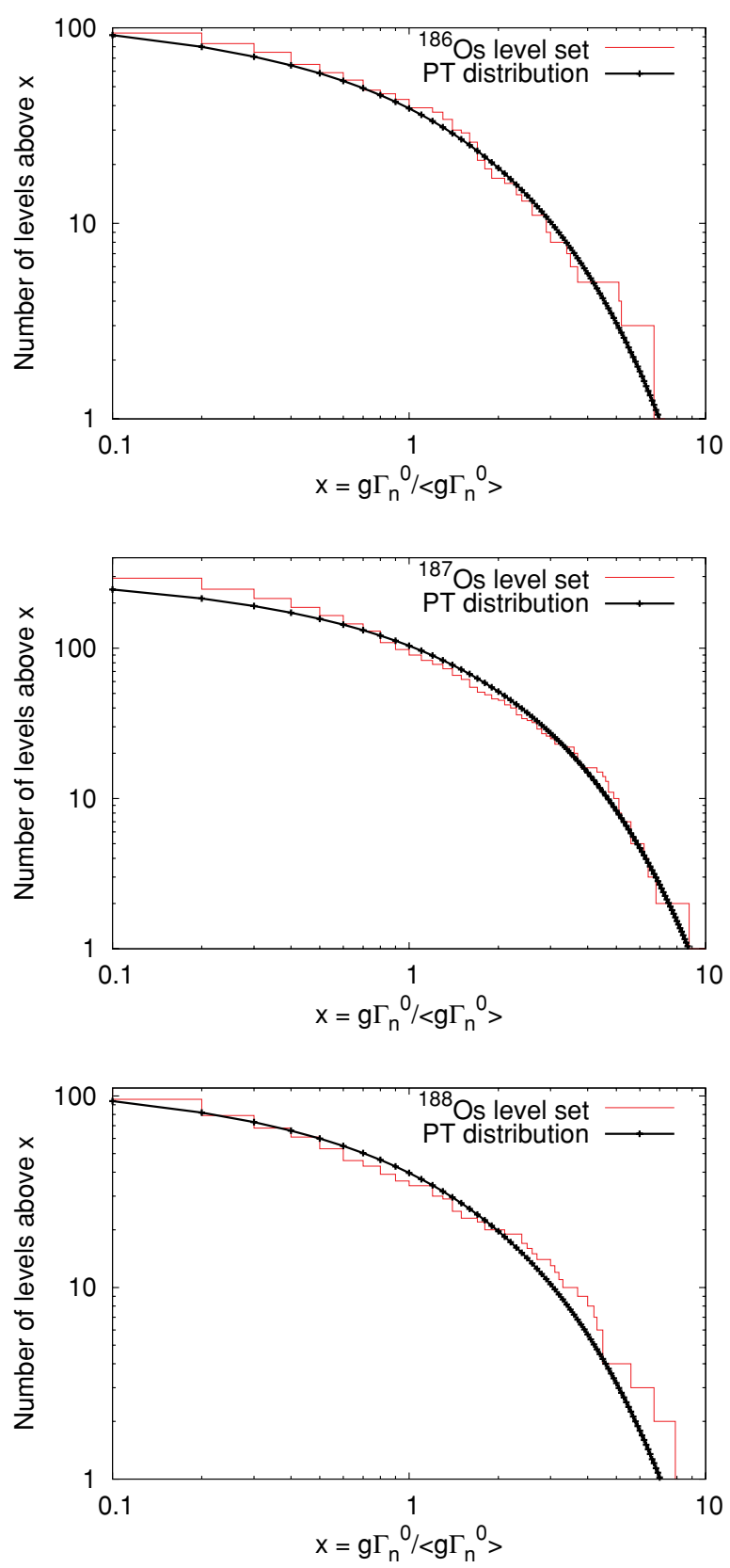

FIG. 4. (Color online) Integrated PT distributions and histograms of the cumulated number of levels with $g \Gamma_{n}^{0} /\left\langle g \Gamma_{n}^{0}\right\rangle>x$.

described in the previous section are important for establishing a consistent parametrization for model calculations of the required stellar $(n, \gamma)$ cross sections. The calculations for the respective ground states can be validated against the experimental cross sections and then extended to include the effect of the excited states. The energy range $0.1 \mathrm{keV} \leqslant E_{n} \leqslant$ $1 \mathrm{MeV}$ for this comparison is completely covered by the high-resolution results of the $\mathrm{n}_{-} \mathrm{TOF}$ measurement presented in Paper I [18].

\section{A. Statistical model calculations}

In the energy and mass range of interest for this work, the Hauser-Feshbach statistical model (HFSM) is perfectly 
TABLE III. Average quantities compared to values in Ref. [25].

\begin{tabular}{|c|c|c|c|c|c|c|}
\hline Sample & \multicolumn{2}{|c|}{${ }^{186} \mathrm{Os}$} & \multicolumn{2}{|c|}{${ }^{187} \mathrm{Os}$} & \multicolumn{2}{|c|}{${ }^{188} \mathrm{Os}$} \\
\hline$\left\langle g \Gamma_{n}^{0}\right\rangle(\mathrm{meV})$ & $6.2 \pm 0.7$ & & $2.0 \pm 0.2$ & & $10.5 \pm 1.5$ & \\
\hline$S_{0}\left(\times 10^{-4}\right)$ & $2.33 \pm 0.32$ & $2.30 \pm 0.32$ & $3.51 \pm 0.29$ & $3.04 \pm 0.35$ & $2.69 \pm 0.36$ & $2.39 \pm 0.36$ \\
\hline$\left\langle\Gamma_{\gamma}\right\rangle(\mathrm{meV})$ & $50.0 \pm 1.5$ & $60 \pm 4$ & $61.0 \pm 1.7$ & $76 \pm 4$ & $52.0 \pm 1.8$ & $82 \pm 4$ \\
\hline
\end{tabular}

suited for the calculation of the neutron induced cross sections for the various reaction channels. The HFSM theory relies on the assumption that the reaction proceeds through the formation of a compound nucleus and that the subsequent decay follows well-established statistical properties. In the case of the Os isotopes this assumption has been verified in the previous section where it was shown that the level spacings follow Wigner distributions and the (reduced) neutron decay widths follow PT distributions. Accordingly, the simple HFSM formula for the neutron capture cross section at incident energy $E_{n}$ holds,

$$
\sigma_{n, \gamma}^{i}\left(E_{n}\right)=\frac{\pi}{k_{n}^{2}} \sum_{J, \pi} g_{J} \frac{\sum_{l s} T_{n, l s} T_{\gamma, J}}{\sum_{l s} T_{n, l s}+\sum_{l s} T_{n^{\prime}, l s}+T_{\gamma, J}} W_{\gamma, J},
$$

where $k_{n}$ is the wave number, $l$ the orbital angular momentum for the neutron-nucleus relative motion, and $g_{J}$ the statistical weighting factor for target nuclei in state $i$. The statistical fluctuation properties of the different reaction channels are accounted for by the factor $W_{\gamma}$.

The calculated stellar MACSs have to be weighted with the temperature-dependent population probabilities of states $i$ defined by

$$
p_{i}=\frac{\left(2 I_{i}+1\right) \mathrm{e}^{-E_{i} / k T}}{\sum_{m}\left(2 I_{m}+1\right) \mathrm{e}^{-E_{m} / k T}},
$$

where $E_{i}$ are the excitation energies of target states populated at temperatures $k T$.

Various HFSM implementations produced so far differ among each other by the different nuclear structure and deexcitation models used for the calculation of the transmission functions for the elastic $\left(T_{n}\right)$, inelastic $\left(T_{n^{\prime}}\right)$, and $\gamma$-ray $\left(T_{\gamma}\right)$ channels entering in Eq. (5). Examples of widely used approaches for nuclear astrophysical applications are those of Holmes et al. [29], Harris [30], and the most recent versions of NON-SMOKER [31], MOST [32], and TALYS [33]. Most of these references include also HFSM computer codes for calculation of reaction cross sections. A repository of parameters and systematics of nuclear structure quantities can be found in the "RIPL" initiative (RIPL-2 [34]).

In general, neutron transmission functions are calculated from the optical model description of the neutron-nucleus interaction. In addition, the probabilities for $\gamma$-ray emission can be deduced from giant dipole resonance excitation (GDR) models, applying time-reversal invariance (detailed balance) and the Brink-Axel hypothesis.

The present calculations of the neutron capture cross sections of ${ }^{186} \mathrm{Os},{ }^{187} \mathrm{Os}$, and ${ }^{188}$ Os were executed with the nuclear reaction code TALYS [33] using the standard optical model parameters (OMP) of Moldauer [35] with the real part of the spherical potential adjusted to reproduce the inelastic cross-section measurement (Paper II) [19]. The level-density parametrization is that of Gilbert and Cameron [36] with level-density parameters $a$, which are adjusted to reproduce the average level spacings $\left\langle D_{0}\right\rangle$ deduced from the present $n_{-}$TOF analysis.

The $\gamma$-ray transmission coefficients were calculated according to the Brink-Axel model with a double-peaked Lorentzian shape $[33,37]$ using the GDR parameters given in Table IV. Since data for ${ }^{187}$ Os were missing, the ones for ${ }^{189}$ Os have been adopted in this case. The average $\gamma$-ray strengths were normalized to reproduce the experimental MACS values at $k T=30 \mathrm{keV}$ [18]. Because of this normalization, the influence of small variations in the GDR parameters is completely negligible.

Additional calculations have been made with a deformed optical potential, considering the coupling of the $0^{+}, 2^{+}, 4^{+}$ rotational states in ${ }^{186}$ Os and ${ }^{188}$ Os and the $1 / 2^{-}, 3 / 2^{-}$, and $5 / 2^{-}$states in ${ }^{187}$ Os. The coupling strengths were deduced from the ground-state quadrupole and octupole deformations, which are also available from the ENSDF data library [38]. Both sets of calculations were normalized to reproduce the MACS at $k T=30 \mathrm{keV}$ (Paper I, Ref. [18]).

These calculations are compared in Fig. 5 with the experimental results obtained at $n_{-}$TOF [18] and in previous measurements [39-41]. One finds that the use of the deformed OMP sets does not provide a better description of the capture cross sections in the energy range of interest compared to the spherical OMP approach. This can be due to an overestimation of the coupling strength deduced from the static quadrupole deformations. In the actual situation, a smaller coupling strength could be more appropriate for the description of the experimental data, a situation comparable to that described by a spherical OMP set. In all cases, the cross sections obtained with the spherical OMP are closer to the experimental data,

TABLE IV. Parameters of the split giant dipole resonance [37]. ${ }^{\mathrm{a}}$

\begin{tabular}{lccc}
\hline \hline & Energy (MeV) & $\Gamma(\mathrm{MeV})$ & $\sigma_{0}(\mathrm{mb})$ \\
\hline${ }^{188} \mathrm{Os}$ & 12.81 & 2.76 & 260 \\
& 14.88 & 4.19 & 390 \\
${ }^{189} \mathrm{Os}^{a}$ & 12.68 & 2.71 & 268 \\
& 14.68 & 3.62 & 395 \\
\hline \hline
\end{tabular}

${ }^{\text {a In our calculations the values for }{ }^{189} \text { Os have also been adopted for }}$ ${ }^{187}$ Os (see text). 

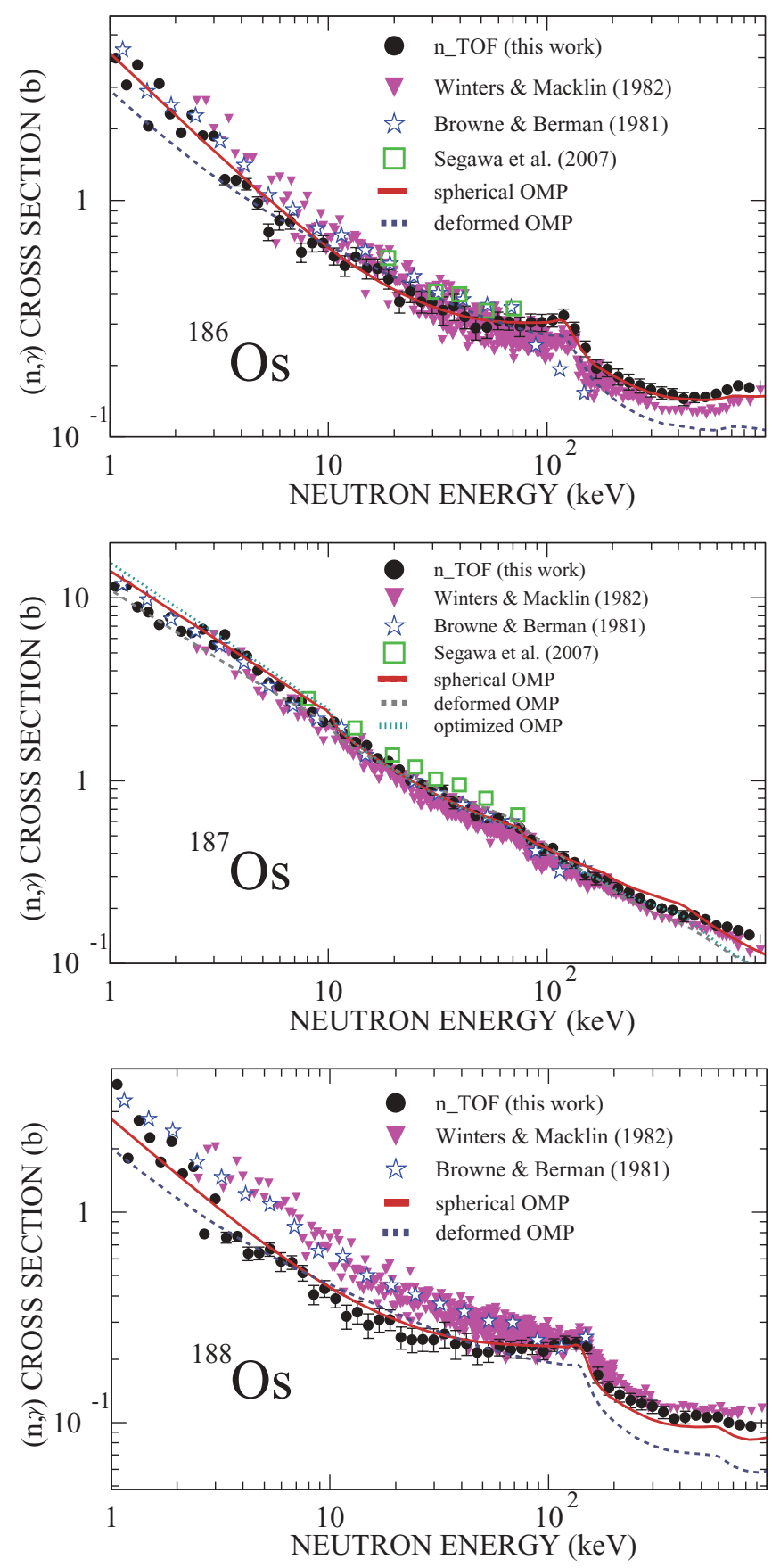

FIG. 5. (Color online) Comparison of measured cross sections with three types of TALYS calculations performed with different OMP sets, the standard values from Moldauer [35], a deformed optical model with channel coupling, and a spherical OMP set with optimized parameters derived from experimental data. The experimental data are best described by the modified parameter set deduced as described in Sec. II.

particularly at the kinks, which mark the opening of inelastic channels.

The preference for the calculations with the present spherical OMP approach is further supported by the plot of the MACS ratio $R_{\sigma}=\left\langle\sigma_{186}\right\rangle /\left\langle\sigma_{187}\right\rangle$ in Fig. 6.

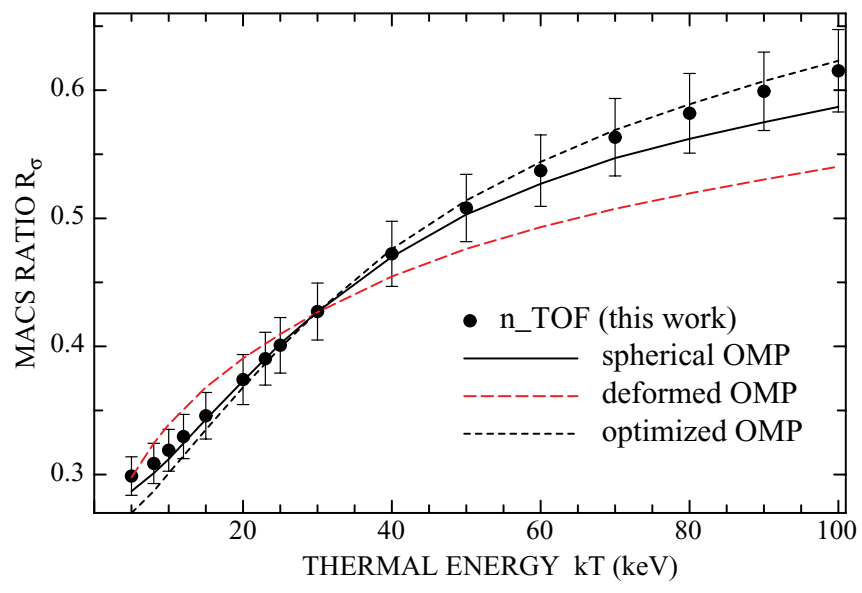

FIG. 6. (Color online) Ratio $\mathrm{MACS}_{186} / \mathrm{MACS}_{187}$ obtained from the measured cross sections (full circles) compared to TALYS calculations with the parameter sets used in Fig. 5.

A similar behavior is also seen in the comparison with the experimental data for the inelastic cross section, where deformed OMP calculations also do no improve the results obtained with spherical OMPs. In fact, the best agreement with the data for the neutron capture as well as for the inelastic channel is found by a small adjustment of the spherical OMPs, i.e., by simply reducing the strength for the real part of the spherical potential from $V_{0}=46$ to $42.5 \mathrm{MeV}$. This modification has little impact on the calculated capture cross section as illustrated in Figs. 5 and 6.

As a conservative estimate of the uncertainties related to the OMPs, the deviation of the cross sections calculated with the three OMP sets from the mean value was adopted in the evaluation of the stellar enhancement factors.

\section{B. Stellar enhancement factors}

The stellar reaction rates that are required to determine the $s$-process abundances of ${ }^{186}$ Os and ${ }^{187}$ Os must be evaluated by due consideration of the possibility that low-lying excited states are populated in the dense thermal photon bath at the stellar site of the $s$ process. Neutron capture rates are sensitive to this effect because the cross sections for excited states may well differ from that of the ground state. The importance of the contributions by excited states to the effective stellar cross section has been discussed in Paper II [19].

The capture cross section of excited states can be modeled as for the ground state. However, there are additional inelastic scattering channels, which have to be considered, i.e., the superelastic channel, where the incident neutron is scattered on an excited state to a lower state in the target. Accordingly, the transmission coefficients $T_{n^{\prime}, l s}$ for the inelastic channels in Eq. (5) must include the open superelastic channels. Comparison of the model calculation with experimental data for the inelastic-scattering cross section provides a good benchmark for the neutron-nucleus interaction used to determine the transmission functions.

As a consequence of the compound nucleus reaction mechanism the compound nuclear states formed by capture on excited states are the same (except for total angular 


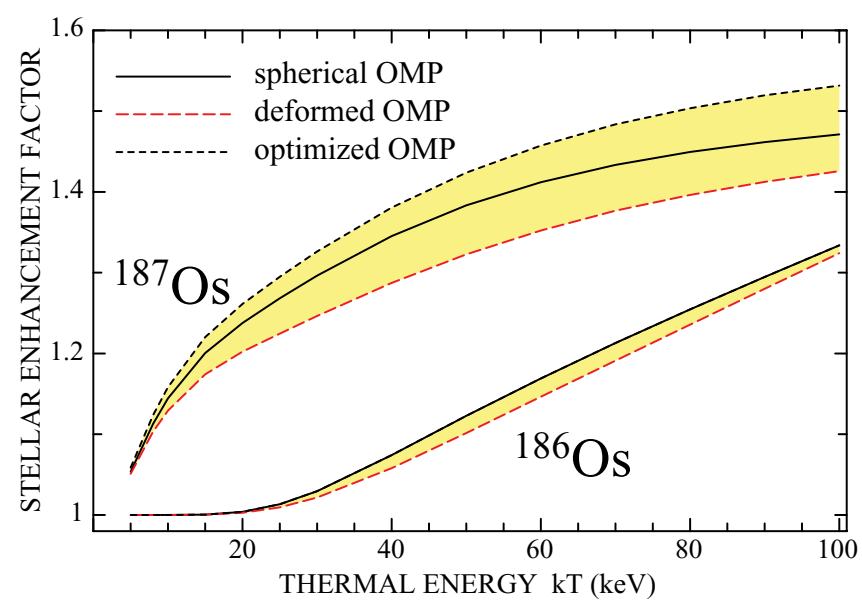

FIG. 7. (Color online) Stellar enhancement factors (SEF) for ${ }^{186} \mathrm{Os}$ and ${ }^{187}$ Os obtained in TALYS calculations with the same OMP sets as used in Fig. 5. The average value at $k T=30 \mathrm{keV}$ has been adopted for the estimate in Fig. 8. The deviations from the mean can be conservatively assumed to represent the associated uncertainties.

momentum) as those populated by capture on the ground state. Because the modeling of the reaction cross section for the ground state can be verified by means of the experimental capture cross sections, the uncertainties of the stellar cross sections are significantly reduced.

The stellar cross section $\langle\sigma\rangle^{*}$ is defined as

$$
\langle\sigma\rangle^{*}=\mathrm{SEF} \times\langle\sigma\rangle^{\mathrm{lab}},
$$

where the SEF factor represents the correction for the contribution by excited states. The MACS for the measured (ground-state) cross section is

$$
\langle\sigma\rangle^{\mathrm{lab}}=\frac{2}{\sqrt{\pi}} \frac{\int_{0}^{\infty} \sigma\left(E_{n}\right) E_{n} \mathrm{e}^{-E_{n} / k T} d E_{n}}{\int_{0}^{\infty} E_{n} \mathrm{e}^{-E_{n} / k T} d E_{n}},
$$

as discussed in Paper I [18].

The SEF corrections are particularly relevant for ${ }^{187}$ Os, where the ground state is populated by only about $30 \%$ at $k T=30 \mathrm{keV}$, while $70 \%$ of the nuclei exist in excited states, $47 \%$ alone in the first excited state at $9.75 \mathrm{keV}$, the state which strongly dominates the competition by inelastic and superelastic scattering. The comparison of the SEF values for ${ }^{186}$ Os and ${ }^{187}$ Os in Fig. 7 underlines the importance of this correction for ${ }^{187} \mathrm{Os}$ in the relevant range of thermal energies around $k T=25 \mathrm{keV}$.

An uncertainty of $\pm 4 \%$ was estimated for the SEF of ${ }^{187} \mathrm{Os}$ at $k T=30 \mathrm{keV}$ from the difference between the results obtained with a spherical and a deformed optical model potential for the neutron-nucleus interaction. This value corresponds to a $17 \%$ uncertainty for the entire contribution of the excited states to the overall cross section. The relatively small uncertainty is justified for the well-studied case of ${ }^{187}$ Os. In general, SEF corrections can be estimated with uncertainties of $\approx 5 \%$ if the capture cross sections for the ground state are known with sufficient accuracy and if the model parameters in the HFSM calculations can be determined from experimental data.

The recommended sets of SEFs for ${ }^{186} \mathrm{Os}$ and ${ }^{187} \mathrm{Os}$ determined in the present calculations are summarized in
TABLE V. Stellar enhancement factors for ${ }^{186}$ Os and ${ }^{187}$ Os.

\begin{tabular}{lcc}
\hline \hline \multirow{2}{*}{$\begin{array}{c}\text { Thermal energy } \\
(\mathrm{keV})\end{array}$} & \multicolumn{2}{c}{ SEF } \\
\cline { 2 - 3 } & $186 \mathrm{Os}$ & ${ }^{187} \mathrm{Os}$ \\
\hline 5 & $1.000 \pm 0.000$ & $1.054 \pm 0.004$ \\
8 & $1.000 \pm 0.000$ & $1.113 \pm 0.011$ \\
10 & $1.000 \pm 0.000$ & $1.144 \pm 0.015$ \\
15 & $1.000 \pm 0.000$ & $1.198 \pm 0.024$ \\
20 & $1.003 \pm 0.001$ & $1.234 \pm 0.032$ \\
25 & $1.012 \pm 0.003$ & $1.263 \pm 0.038$ \\
30 & $1.027 \pm 0.005$ & $1.290 \pm 0.043$ \\
40 & $1.069 \pm 0.011$ & $1.338 \pm 0.050$ \\
50 & $1.116 \pm 0.014$ & $1.377 \pm 0.054$ \\
60 & $1.162 \pm 0.015$ & $1.407 \pm 0.055$ \\
70 & $1.206 \pm 0.015$ & $1.431 \pm 0.055$ \\
80 & $1.248 \pm 0.013$ & $1.450 \pm 0.054$ \\
90 & $1.290 \pm 0.010$ & $1.465 \pm 0.055$ \\
100 & $1.331 \pm 0.007$ & $1.476 \pm 0.056$ \\
\hline \hline
\end{tabular}

Table V. The corresponding MACS values for the ground state obtained from the experimental $(n, \gamma)$ cross sections are summarized in Table III of Paper I [18].

\section{TUNING THE CLOCK}

The implications of the different uncertainties of the Re/Os cosmochronometer are illustrated by their impact on the time duration of nucleosynthesis and consequently on the galactic age using the schematic model proposed by Fowler and Hoyle that assumes an exponential decrease of element production by the $r$ process, which is commonly associated with supernovae $[7,42]$.

Application of this model to the Re/Os cosmochronometer provides a straightforward way to study the impact of the uncertainties related to the nuclear physics involved. For an exponentially decreasing production rate of ${ }^{187} \mathrm{Re}$, the abundance ratio ${ }^{187} \mathrm{Os} /{ }^{187} \mathrm{Re}$ evolves with time as

$$
\frac{N_{\mathrm{c}}\left({ }^{187} \mathrm{Os}\right)}{N\left({ }^{187} \mathrm{Re}\right)}=\left[\frac{\Lambda-\lambda}{\Lambda} \mathrm{e}^{\Lambda t_{0}} \frac{1-\mathrm{e}^{-\Lambda t_{0}}}{1-\mathrm{e}^{-(\Lambda-\lambda) t_{0}}}\right]-1,
$$

where $\Lambda$ and $t_{0}$ stand for the time constant and duration of $r$-process nucleosynthesis, $\lambda$ for the decay rate of ${ }^{187} \mathrm{Re}$, and ${ }^{187}$ Os for the radiogenic part of the isotopic abundance defined in Eq. (1) [7]. The present MACS values have been used for deriving the radiogenic component of ${ }^{187} \mathrm{Os}$ in order to minimize systematic uncertainties in the cross section ratio $\langle\sigma\rangle_{186} /\langle\sigma\rangle_{187}$ as described in Sec. V of Paper I [18].

In Eq. (9), corrections of the ${ }^{187} \mathrm{Re}$ and ${ }^{187} \mathrm{Os}$ abundances due to the $s$-process branchings at ${ }^{185} \mathrm{~W}$ and ${ }^{186} \mathrm{Re}$ (Fig. 1) and due to the $p$ process are not yet included. The $p$-process component of the ${ }^{187}$ Os abundance may well be negligible in view of the very rare $p$-only isotope ${ }^{184} \mathrm{Re}$, but the correction for the $s$-process branchings must be considered in a full analysis in the context of a galactic chemical evolution model.

Nevertheless, the simplified approach expressed via Eq. (9) can be used to illustrate the basic idea of the Re/Os clock. The evolution of the abundance ratio $\frac{N_{\mathrm{c}}\left({ }^{187} \mathrm{Os}\right)}{N\left({ }^{187} \mathrm{Re}\right)}$ is plotted in Fig. 8 


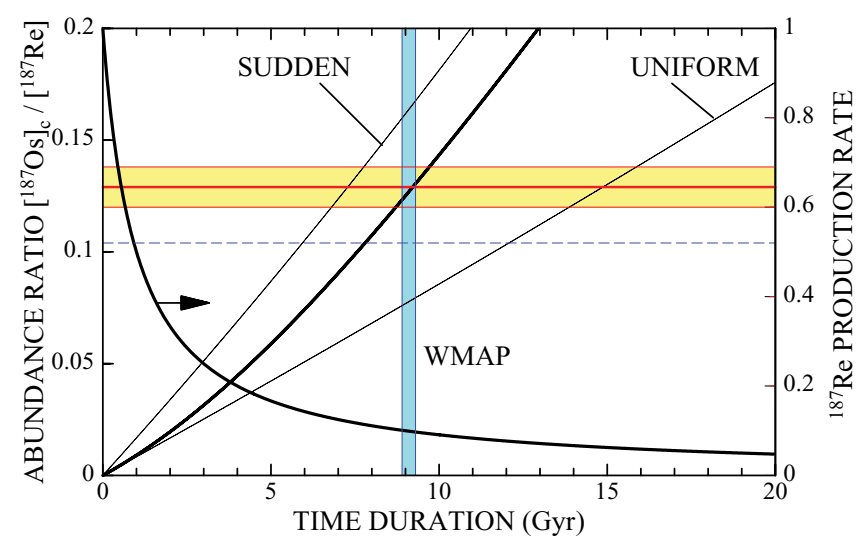

FIG. 8. (Color online) Time evolution of the abundance ratio of ${ }^{187} \mathrm{Os}$ and ${ }^{187} \mathrm{Re}$ obtained with the schematic model represented by Eq. (9) for different time constants $\Lambda$ of $r$-process nucleosynthesis. The abundance ratio determined by the data in Table VI is indicated by the shaded horizontal bar. The thick solid lines refer to a solution with $\Lambda=0.5 \mathrm{Gyr}^{-1}$, which matches the age derived from WMAP (vertical bar) [44]. The effect of the SEFs is illustrated by the dashed line, where these corrections have been omitted.

for different assumptions concerning the history of $r$-process nucleosynthesis before the formation of the solar system 4.55 Gyr ago. The two extreme conditions for the production rate of ${ }^{187} \mathrm{Re}$ corresponding to time constants $\Lambda \Rightarrow 0$ and $\Lambda \Rightarrow \infty$ lead to the cases of a sudden origin at the beginning of the galaxy and a uniform production with time.

The horizontal bar in Fig. 8 indicates the ratio obtained with the input data listed in Table VI, which restrains the age between 7 and $15 \mathrm{Gyr}$ according to the extreme assumptions for the production rate. The exponential solution marked by thick by solid lines in Fig. 8, which corresponds to a time constant $\Lambda=0.5 \mathrm{Gyr}^{-1}$, was chosen to reproduce the age claimed by the Wilkinson Microwave Anisotropy Probe (WMAP) collaboration [44].

The main purpose of Fig. 8 is, however, to illustrate the important effect of accurate neutron cross sections. The present improvement of the $(n, \gamma)$ cross sections and of the SEFs implies a reduced uncertainty in the time duration of nucleosynthesis, $t_{0}$, of about 0.5 Gyr. The SEF correction itself affects the clock by $1.5 \mathrm{Gyr}$ as indicated by the dashed horizontal line.
A complete picture of the various nuclear physics components and of the related uncertainties (including the observed abundances) is summarized in Table VI. The full set adds up to less than 1 Gyr uncertainty in the age. At this point, the remaining relevant uncertainties of the $\mathrm{Re} / \mathrm{Os}$ clock are all related to astrophysical rather than nuclear issues.

The first astrophysical issue concerns the effect of astration describing the fate of ${ }^{187} \mathrm{Re}$ going through different stellar generations. Because of the strong temperature dependence of its half-life, ${ }^{187} \mathrm{Re}$ can be partly destroyed in this way. Another important aspect concerns the time dependence of the production rate, which has to be treated in the framework of an appropriate galactic chemical evolution model including a detailed calculation of the $s$-process abundance components.

In the light of the improved nuclear physics input, it appears rewarding to address the astrophysical part of the $\mathrm{Re} / \mathrm{Os}$ clock with new interest. Current progress in stellar modeling and galactic chemical evolution may well provide an extended and consistent analysis of the Re/Os age, which would be a most valuable complement to other cosmochronometers such as the Hubble age [45], the age of globular clusters [46], and the age deduced from the cosmic microwave background [47] and from the $\mathrm{U} / \mathrm{Th}$ abundances in extremely metal-poor halo stars [4-6]. While each of these chronometers has intrinsic problems by itself, their combination will provide a much firmer value for the age of the universe.

The different features of the radioactive dating methods based on ${ }^{187} \mathrm{Re}$ and $\mathrm{U} / \mathrm{Th}$ can be considered as an example for this expectation. While the nuclear part is well understood, the persisting problem of the $\mathrm{Re} / \mathrm{Os}$ clock is related to the time dependence of galactic evolution $[10,11]$. Contrary, extremely metal-poor stars were formed very early in the galaxy and their present $\mathrm{U} / \mathrm{Th}$ ratio is the result of free decay in a well-defined closed system, undisturbed by chemical evolution effects. In this case, however, the difficulty resides in the nucleosynthesis part, because the initial $\mathrm{U} / \mathrm{Th}$ abundances are determined by the large nuclear physics uncertainties on the $r$-process path that runs close to the neutron drip line. Therefore, the combined analysis of both clocks may provide important constraints for galactic chemical evolution.

\section{SUMMARY}

The neutron capture cross sections of ${ }^{186,187,188}$ Os, which were measured at CERN n_TOF [18], have been analyzed in

TABLE VI. The uncertainties of the Re/Os cosmochronometer associated with the Re/Os abundance ratios and the nuclear physics input.

\begin{tabular}{lcc}
\hline \hline Quantity & Value & Uncertainty for galactic age (Gyr) \\
\hline${ }^{186} \mathrm{Os} /{ }^{187} \mathrm{Re}$ abundance ratio ${ }^{\mathrm{a}}$ & $0.2845 \pm 0.0071$ & Reference \\
${ }^{187} \mathrm{Os} /{ }^{187} \mathrm{Re}$ abundance ratio & \\
Total effect of abundances & $0.2254 \pm 0.0057$ & 0.49 \\
$t_{1 / 2}\left({ }^{187} \mathrm{Re}\right)$ & & 0.29 \\
$\mathrm{MACS}-30$ ratio $R_{\sigma}^{\mathrm{lab}}$ & $41.2 \pm 1.1(\mathrm{Gyr})$ & 0.40 \\
$\mathrm{SEF}$ of ${ }^{186}$ Os at $k T=30 \mathrm{keV}$ & $0.427 \pm 0.023$ & 0.04 \\
SEF of ${ }^{187}$ Os at $k T=30 \mathrm{keV}$ & $1.027 \pm 0.005$ & 0.24 \\
Total & $1.29 \pm 0.04$ & 0.74 \\
\hline \hline
\end{tabular}

${ }^{\mathrm{a}}$ At the time of solar system formation. 
the resolved resonance region with the $R$-matrix code SAMMY. In total 186, 480, and 199 resonances were identified for these isotopes between 5.0, 3.0, and $8.0 \mathrm{keV}$, respectively. From these results average level spacings, radiative widths, and neutron strength functions have been deduced by statistical analyses to establish a consistent set of input data for detailed cross section calculations with the Hauser-Feshbach statistical model. Based on these calculations stellar enhancement factors were obtained to correct the Maxwellian averaged cross sections determined from experimental $(n, \gamma)$ data for the effect of thermally excited states in the hot, dense photon bath at the $s$-process site. The corresponding stellar $(n, \gamma)$ cross sections have been used to separate the radiogenic part of the ${ }^{187} \mathrm{Os}$ abundance from its $s$-process component and to define the mother/daughter ratio ${ }^{187} \mathrm{Re} /{ }^{187}$ Os. With a schematic model that assumes an exponentially decreasing production rate for ${ }^{187} \mathrm{Re}$, it was shown that the remaining nuclear physics uncertainties affect the age obtained by the Re/Os clock by less than $1 \mathrm{Gyr}$.

\section{ACKNOWLEDGMENTS}

This work was supported by the EC under contract FIKW-CT-2000-00107 and by the funding agencies of the participating institutes.

\section{APPENDIX}

The resonance parameters deduced from the present data are listed for ${ }^{186} \mathrm{Os},{ }^{187} \mathrm{Os}$, and ${ }^{188} \mathrm{Os}$ in Tables VII-IX, respectively.

TABLE VII. Resonance parameters of ${ }^{186}$ Os.

\begin{tabular}{|c|c|c|c|}
\hline$E_{r}(\mathrm{eV})$ & $\Gamma_{\gamma}(\mathrm{meV})$ & $g \Gamma_{n}(\mathrm{meV})$ & $\frac{g \Gamma_{\gamma} \Gamma_{n}}{\Gamma}(\mathrm{meV})$ \\
\hline \multicolumn{4}{|l|}{$22.28(0)$} \\
\hline \multicolumn{4}{|l|}{$44.80(0)$} \\
\hline \multicolumn{4}{|l|}{$66.25(0)$} \\
\hline \multicolumn{4}{|l|}{$89.72(0)$} \\
\hline $108.83(1)$ & $50.0(0)$ & $0.35(1)$ & $0.35(1)$ \\
\hline $137.886(3)$ & $41.7(3)$ & $435.0(0)$ & $38.1(3)$ \\
\hline $145.28(1)$ & $50.0(0)$ & $1.5(1)$ & $1.50(6)$ \\
\hline $169.797(2)$ & $50.0(0)$ & $5.32(5)$ & 4.81(4) \\
\hline 201.995(3) & & & $34.8(3)$ \\
\hline $249.642(3)$ & $50.0(0)$ & $13.5(2)$ & $10.6(1)$ \\
\hline $273.10(1)$ & $50.0(0)$ & $2.08(4)$ & $2.00(4)$ \\
\hline 281.21(1) & & & $33.0(6)$ \\
\hline $313.531(4)$ & & & $24.4(6)$ \\
\hline $342.80(2)$ & $42.9(6)$ & $673.8(0)$ & $40.3(6)$ \\
\hline $370.14(1)$ & & & $33.4(8)$ \\
\hline $380.25(1)$ & & & $30.3(8)$ \\
\hline 417.06(1) & & & $25.5(9)$ \\
\hline $423.39(1)$ & & & 29.2(7) \\
\hline $452.32(1)$ & $50.0(0)$ & $19.6(4)$ & $14.1(3)$ \\
\hline $521.36(1)$ & & & $42.3(8)$ \\
\hline $568.26(1)$ & $32.4(5)$ & $243.1(0)$ & $28.6(4)$ \\
\hline $604.93(1)$ & & & $38.0(10)$ \\
\hline $635.17(1)$ & & & $42.5(9)$ \\
\hline $643.95(2)$ & $39.8(7)$ & $509.0(0)$ & $36.9(7)$ \\
\hline
\end{tabular}

TABLE VII. (Continued.)

\begin{tabular}{|c|c|c|c|}
\hline$E_{r}(\mathrm{eV})$ & $\Gamma_{\gamma}(\mathrm{meV})$ & $g \Gamma_{n}(\mathrm{meV})$ & $\frac{g \Gamma_{\gamma} \Gamma_{n}}{\Gamma}(\mathrm{meV})$ \\
\hline $655.66(1)$ & $40.5(8)$ & $462.2(0)$ & $37.3(7)$ \\
\hline $680.16(2)$ & $46.8(8)$ & $342.1(0)$ & $41.2(7)$ \\
\hline $736.35(2)$ & $50.0(0)$ & 13.7(4) & $10.7(3)$ \\
\hline $763.50(1)$ & & & $38.9(18)$ \\
\hline 797.17(1) & & & 25.1(10) \\
\hline $837.71(2)$ & $43.8(8)$ & $362.0(0)$ & $39.1(7)$ \\
\hline $846.18(1)$ & $50.0(0)$ & 37.2(13) & $21.3(7)$ \\
\hline $869.15(4)$ & $38.1(9)$ & $724.5(0)$ & $36.2(8)$ \\
\hline 891.71(1) & & & $42.7(13)$ \\
\hline $916.01(3)$ & $50.0(0)$ & $5.8(3)$ & $5.18(23)$ \\
\hline $962.84(7)$ & $43.6(11)$ & $1611(0)$ & $42.4(11)$ \\
\hline 980.83(3) & $41.5(11)$ & 819.3(0) & $39.5(11)$ \\
\hline $1024.38(2)$ & & & $44.3(7)$ \\
\hline 1038.04(2) & & & $41.4(7)$ \\
\hline 1073.72(2) & $49.3(14)$ & $73.0(0)$ & $29.4(8)$ \\
\hline 1106.31(1) & $50.0(0)$ & $6.6(11)$ & $5.8(10)$ \\
\hline 1171.06(2) & $50.0(0)$ & $19.1(6)$ & $13.8(4)$ \\
\hline 1205.01(2) & & & $22.1(8)$ \\
\hline $1216.03(6)$ & $50.0(0)$ & $4.7(3)$ & $4.27(24)$ \\
\hline $1224.58(2)$ & & & $21.8(9)$ \\
\hline $1228.76(3)$ & $36.5(9)$ & $556.1(0)$ & $34.2(8)$ \\
\hline $1244.27(1)$ & & & $31.5(15)$ \\
\hline $1264.70(2)$ & & & $26.7(11)$ \\
\hline 1298.61(3) & $55.0(10)$ & $855.0(0)$ & $51.6(9)$ \\
\hline $1324.42(2)$ & & & $41.0(9)$ \\
\hline 1344.79(1) & $50.0(0)$ & $5.5(10)$ & $5.0(10)$ \\
\hline 1359.99(6) & $56.2(13)$ & 1750.(0) & $54.4(12)$ \\
\hline $1402.92(6)$ & $50.0(0)$ & $5.1(3)$ & 4.7(3) \\
\hline 1405.29(1) & $50.0(0)$ & 4.4(8) & $4.0(8)$ \\
\hline $1462.99(2)$ & $45.9(9)$ & $311.0(0)$ & $40.0(8)$ \\
\hline $1476.90(2)$ & & & $40.4(19)$ \\
\hline $1504.44(2)$ & $54.5(12)$ & $169.0(0)$ & $41.2(10)$ \\
\hline 1529.16(1) & $50.0(0)$ & $13.0(21)$ & $10.3(17)$ \\
\hline $1559.55(4)$ & $50.0(0)$ & 10.1(4) & $8.4(4)$ \\
\hline 1597.45(3) & 47.9(13) & $139.0(0)$ & $35.6(10)$ \\
\hline $1626.87(3)$ & $53.3(11)$ & $489.0(0)$ & $48.1(10)$ \\
\hline $1674.85(1)$ & $50.0(0)$ & $108(16)$ & $34.1(51)$ \\
\hline 1687.37(2) & & & $28.2(27)$ \\
\hline 1713.66(3) & $50.0(0)$ & $168.0(0)$ & $38.5(0)$ \\
\hline $1742.42(3)$ & $50.0(0)$ & 49.9(76) & $25.0(38)$ \\
\hline $1748.28(3)$ & $43.9(12)$ & $413.1(0)$ & $39.7(11)$ \\
\hline $1790.23(2)$ & $50.0(0)$ & 15.7(30) & $11.9(23)$ \\
\hline 1797.56(4) & $50.0(0)$ & $23.7(11)$ & $16.1(7)$ \\
\hline 1821.70(3) & & & $34.3(34)$ \\
\hline 1860.11(3) & & & $40.8(13)$ \\
\hline 1876.08(3) & $50.0(0)$ & $3.6(7)$ & $3.4(7)$ \\
\hline 1919.51(3) & $53.4(17)$ & $113.0(0)$ & $36.2(11)$ \\
\hline 1936.88(4) & $50.0(0)$ & 29.1(15) & $18.4(9)$ \\
\hline $1957.36(2)$ & $45.4(17)$ & $253.0(0)$ & $38.5(14)$ \\
\hline 1971.87(10) & $50.0(0)$ & $5.4(4)$ & $4.9(4)$ \\
\hline 1991.27(5) & $38.8(12)$ & $383.0(0)$ & $35.2(11)$ \\
\hline 2018.32(6) & $50.0(0)$ & $11.5(7)$ & $9.3(5)$ \\
\hline 2065.90(4) & $56.7(14)$ & $304.0(0)$ & $47.8(12)$ \\
\hline 2091.47(4) & $51.6(15)$ & $236.0(0)$ & $42.3(12)$ \\
\hline 2137.45(7) & $50.0(0)$ & 7.8(10) & $6.8(9)$ \\
\hline
\end{tabular}


TABLE VII. (Continued.)

\begin{tabular}{|c|c|c|c|}
\hline$E_{r}(\mathrm{eV})$ & $\Gamma_{\gamma}(\mathrm{meV})$ & $g \Gamma_{n}(\mathrm{meV})$ & $\frac{g \Gamma_{\gamma} \Gamma_{n}}{\Gamma}(\mathrm{meV})$ \\
\hline 2167.41(4) & $55.1(17)$ & $148.0(0)$ & $40.1(12)$ \\
\hline 2186.45(1) & $50.0(0)$ & $7.5(15)$ & $6.5(13)$ \\
\hline $2201.59(5)$ & $50.0(0)$ & $73.0(0)$ & $29.7(0)$ \\
\hline $2221.28(5)$ & $50.0(0)$ & $78.0(0)$ & $30.5(0)$ \\
\hline $2229.25(5)$ & & & $40.7(37)$ \\
\hline $2273.50(1)$ & & & $24.8(42)$ \\
\hline 2301.71(6) & $53.3(18)$ & $475.0(0)$ & $48.0(17)$ \\
\hline $2309.52(5)$ & $52.3(20)$ & $226.0(0)$ & $42.4(16)$ \\
\hline $2313.20(5)$ & $45.6(18)$ & $207.0(0)$ & $37.4(15)$ \\
\hline 2373.94(5) & $53.1(15)$ & $635.0(0)$ & $49.0(14)$ \\
\hline 2391.11(3) & $48.9(16)$ & $320.0(0)$ & $42.4(14)$ \\
\hline 2432.34(1) & $50.0(0)$ & 5.1(10) & $4.6(9)$ \\
\hline 2439.71(6) & $45.8(16)$ & $996.0(0)$ & $43.8(15)$ \\
\hline $2473.25(5)$ & $56.4(20)$ & $242.0(0)$ & $45.8(16)$ \\
\hline $2489.42(1)$ & & & $28.8(47)$ \\
\hline $2538.42(6)$ & $50.0(0)$ & $94.0(0)$ & $32.4(0)$ \\
\hline 2586.98(6) & $50.3(16)$ & $651.0(0)$ & $46.7(15)$ \\
\hline $2597.51(10)$ & $50.0(0)$ & $12.0(9)$ & $9.7(7)$ \\
\hline $2626.71(8)$ & & & $30.5(37)$ \\
\hline $2655.50(7)$ & & & $25.7(22)$ \\
\hline $2660.57(8)$ & & & $44.7(50)$ \\
\hline $2666.76(1)$ & $50.0(0)$ & $8.5(16)$ & $7.3(14)$ \\
\hline 2733.89(6) & $56.9(18)$ & $341.0(0)$ & $48.7(16)$ \\
\hline $2748.05(7)$ & & & $37.3(50)$ \\
\hline $2765.52(6)$ & & & $35.2(37)$ \\
\hline $2807.33(6)$ & $60.5(20)$ & $765.0(0)$ & 56.1(19) \\
\hline $2821.50(6)$ & $62.7(22)$ & $471.0(0)$ & $55.3(20)$ \\
\hline $2852.17(6)$ & & & $31.3(60)$ \\
\hline 2882.23(6) & $60.4(25)$ & $167.0(0)$ & $44.4(19)$ \\
\hline 2907.66(9) & $53.4(19)$ & $994.0(0)$ & $50.7(18)$ \\
\hline $2935.10(7)$ & & & $37.0(48)$ \\
\hline 2941.75(3) & $50.0(0)$ & $10.3(20)$ & $8.6(17)$ \\
\hline $2960.70(7)$ & $55.2(30)$ & $145(27)$ & $39.9(79)$ \\
\hline $2968.82(6)$ & $47.6(20)$ & $275.0(0)$ & $40.6(17)$ \\
\hline 2990.61(8) & & & $30.1(20)$ \\
\hline $3036.00(10)$ & & & $17.8(14)$ \\
\hline $3059.97(6)$ & $51.8(21)$ & $179.0(0)$ & $40.2(16)$ \\
\hline $3102.31(7)$ & 51.1(19) & $359.0(0)$ & $44.7(16)$ \\
\hline $3118.60(7)$ & $45.1(18)$ & $235.0(0)$ & $37.9(15)$ \\
\hline 3138.71(7) & $49.0(20)$ & $235.0(0)$ & $40.6(16)$ \\
\hline $3197.47(2)$ & $53(10)$ & 20.9(41) & $15.0(41)$ \\
\hline $3207.88(7)$ & $55.2(25)$ & $231.0(0)$ & $44.6(20)$ \\
\hline $3218.33(6)$ & & & $45.1(20)$ \\
\hline $3250.78(23)$ & $50.0(0)$ & 2690.(0) & $49.1(0)$ \\
\hline $3317.97(9)$ & & & $38.1(56)$ \\
\hline $3338.33(8)$ & $58.5(25)$ & $268.0(0)$ & $48.0(20)$ \\
\hline $3356.35(13)$ & $49.7(22)$ & 1230.(0) & $47.8(21)$ \\
\hline $3419.15(1)$ & & & $14.9(29)$ \\
\hline $3434.51(11)$ & & & $22.8(19)$ \\
\hline $3460.63(9)$ & & & $44.2(70)$ \\
\hline 3489.93(8) & & & $31.6(56)$ \\
\hline $3510.80(8)$ & & & $44.6(70)$ \\
\hline $3521.57(23)$ & & & $8.3(9)$ \\
\hline $3537.65(11)$ & & & $48.8(0)$ \\
\hline $3549.05(11)$ & & & $39.1(57)$ \\
\hline
\end{tabular}

TABLE VII. (Continued.)

\begin{tabular}{|c|c|c|}
\hline$E_{r}(\mathrm{eV})$ & $g \Gamma_{n}(\mathrm{meV})$ & $\frac{g \Gamma_{\gamma} \Gamma_{n}}{\Gamma}(\mathrm{meV})$ \\
\hline 3579.68(9) & & $35.0(44)$ \\
\hline $3647.69(13)$ & & $47.4(0)$ \\
\hline $3662.57(11)$ & & $40.3(76)$ \\
\hline $3681.45(13)$ & & 47.1(64) \\
\hline $3687.20(3)$ & & $14.8(29)$ \\
\hline $3690.54(2)$ & & $31.4(59)$ \\
\hline $3725.29(10)$ & & $35.2(41)$ \\
\hline $3742.40(10)$ & & $47.1(0)$ \\
\hline 3793.53(1) & & $33.6(65)$ \\
\hline $3807.93(20)$ & & $34.5(62)$ \\
\hline $3847.18(12)$ & & $43.2(80)$ \\
\hline $3857.62(26)$ & & $8.0(13)$ \\
\hline $3866.14(10)$ & & $21.2(36)$ \\
\hline $3883.99(10)$ & & $41.7(67)$ \\
\hline $3925.83(1)$ & & $40.7(64)$ \\
\hline 3978.06(1) & & $16.0(32)$ \\
\hline $3995.47(13)$ & & $31.8(38)$ \\
\hline $4015.38(5)$ & & $45.0(72)$ \\
\hline $4026.69(14)$ & & $47.0(67)$ \\
\hline $4055.59(20)$ & & $11.0(19)$ \\
\hline $4071.20(15)$ & & $44.0(83)$ \\
\hline $4085.46(12)$ & & $41.4(65)$ \\
\hline 4142.69(13) & & $43.3(73)$ \\
\hline $4191.41(14)$ & & $30.0(36)$ \\
\hline $4236.33(15)$ & & $47.2(0)$ \\
\hline $4264.83(5)$ & & $19.8(35)$ \\
\hline $4296.68(15)$ & & $43.5(76)$ \\
\hline $4325.54(12)$ & & $44.3(77)$ \\
\hline $4342.79(17)$ & & $46.4(81)$ \\
\hline $4376.71(2)$ & & $42.0(70)$ \\
\hline $4401.94(24)$ & & $15.7(19)$ \\
\hline $4414.62(16)$ & & $42.6(78)$ \\
\hline $4424.65(16)$ & & $41.6(70)$ \\
\hline $4453.98(16)$ & & $35.7(52)$ \\
\hline 4494.04(16) & & $38.1(57)$ \\
\hline $4523.00(1)$ & & $28.2(54)$ \\
\hline $4539.57(25)$ & & $35.7(58)$ \\
\hline $4579.67(16)$ & & $44.6(84)$ \\
\hline $4630.12(24)$ & & $44.9(91)$ \\
\hline 4632.61(18) & & $44.0(73)$ \\
\hline $4648.24(11)$ & & $43.7(79)$ \\
\hline $4673.69(2)$ & & $11.0(22)$ \\
\hline $4695.93(18)$ & & $42.6(88)$ \\
\hline $4706.95(15)$ & & $42.7(77)$ \\
\hline $4747.00(22)$ & & $48.6(59)$ \\
\hline $4778.82(7)$ & & $40.3(64)$ \\
\hline 4846.20(19) & & $45.4(79)$ \\
\hline 4881.59(19) & & $45.2(78)$ \\
\hline $4892.19(15)$ & & $42.8(75)$ \\
\hline $4917.28(5)$ & & $36.6(69)$ \\
\hline $4967.86(6)$ & & $38.0(67)$ \\
\hline 4983.79(18) & & $45.5(75)$ \\
\hline
\end{tabular}

All spins are defined as $s$-wave. Parameters with zero uncertainty were treated as fixed values in the SAMMY fits. 
TABLE VIII. Resonance parameters of ${ }^{187}$ Os.

\begin{tabular}{|c|c|c|c|}
\hline$E_{r}(\mathrm{eV})$ & $\Gamma_{\gamma}(\mathrm{meV})$ & $g \Gamma_{n}(\mathrm{meV})$ & $\frac{g \Gamma_{\gamma} \Gamma_{n}}{\Gamma}(\mathrm{meV})$ \\
\hline \multicolumn{4}{|l|}{$9.479(0)$} \\
\hline \multicolumn{4}{|l|}{$12.700(0)$} \\
\hline \multicolumn{4}{|l|}{$20.230(0)$} \\
\hline 26.351(1) & $61.0(0)$ & $0.25(0)$ & $0.25(0)$ \\
\hline $28.322(2)$ & $61.0(0)$ & $0.15(0)$ & $0.15(0)$ \\
\hline $39.550(0)$ & $61.0(0)$ & $0.91(1)$ & $0.89(1)$ \\
\hline $40.567(1)$ & $61.0(0)$ & $10.27(4)$ & $6.14(2)$ \\
\hline $43.455(1)$ & $61.0(0)$ & $11.07(5)$ & $6.41(3)$ \\
\hline $47.815(1)$ & $61.0(0)$ & $12.51(0)$ & $6.87(0)$ \\
\hline $50.160(0)$ & $61.0(0)$ & $25.00(0)$ & $9.47(0)$ \\
\hline $50.600(0)$ & $61.0(0)$ & $1.95(0)$ & $1.87(0)$ \\
\hline $62.188(1)$ & $61.0(0)$ & $4.50(0)$ & $3.47(0)$ \\
\hline $63.846(1)$ & & & $7.36(6)$ \\
\hline $65.092(2)$ & $61.0(0)$ & $1.00(1)$ & $0.98(1)$ \\
\hline $71.467(2)$ & $61.0(0)$ & $0.77(1)$ & $0.76(1)$ \\
\hline $78.050(0)$ & $61.0(0)$ & $1.88(0)$ & $1.80(0)$ \\
\hline $83.360(0)$ & $61.0(0)$ & $0.53(1)$ & $0.52(1)$ \\
\hline $89.975(1)$ & & & $14.4(3)$ \\
\hline $92.897(1)$ & $61.0(0)$ & $11.19(9)$ & $6.45(5)$ \\
\hline $99.333(2)$ & & & $8.72(15)$ \\
\hline $105.004(2)$ & $61.0(0)$ & 7.07(9) & $6.13(8)$ \\
\hline $108.853(1)$ & & & $32.25(34)$ \\
\hline $110.378(5)$ & $61.0(0)$ & $1.27(2)$ & $1.24(2)$ \\
\hline $114.695(3)$ & $61.0(0)$ & $0.90(1)$ & $0.88(1)$ \\
\hline $118.980(13)$ & $61.0(0)$ & $0.15(1)$ & $0.15(1)$ \\
\hline $123.060(0)$ & $61.0(0)$ & $4.34(5)$ & $3.97(4)$ \\
\hline $124.416(2)$ & & & $12.00(15)$ \\
\hline $126.944(1)$ & & & $24.61(0)$ \\
\hline $132.153(6)$ & $61.0(0)$ & $0.56(1)$ & $0.55(1)$ \\
\hline $138.281(2)$ & $54.4(4)$ & $122.1(0)$ & $30.6(2)$ \\
\hline $145.076(2)$ & & & $24.1(2)$ \\
\hline $155.446(2)$ & $61.0(0)$ & $6.06(6)$ & $5.35(5)$ \\
\hline $164.349(2)$ & $61.0(0)$ & $16.7(2)$ & $12.2(2)$ \\
\hline $168.371(2)$ & $61.0(0)$ & $11.2(1)$ & 8.98(9) \\
\hline 171.311(2) & $61.0(0)$ & $2.75(4)$ & $2.33(3)$ \\
\hline $175.748(2)$ & $61.0(0)$ & $10.9(2)$ & 8.79(12) \\
\hline $176.985(3)$ & & & $28.2(10)$ \\
\hline $178.418(3)$ & $61.0(0)$ & $8.49(9)$ & $7.16(8)$ \\
\hline $188.942(2)$ & $61.0(0)$ & $9.2(1)$ & $7.64(8)$ \\
\hline $196.807(26)$ & $61.0(0)$ & $0.24(2)$ & $0.24(2)$ \\
\hline $201.005(3)$ & $61.0(0)$ & $11.2(1)$ & $9.01(12)$ \\
\hline 207.003(4) & $61.0(0)$ & $27.5(0)$ & $9.81(0)$ \\
\hline $211.469(4)$ & $61.0(0)$ & $6.3(1)$ & $5.53(9)$ \\
\hline $213.366(13)$ & $61.0(0)$ & 206.3(0) & $14.20(0)$ \\
\hline 213.591(10) & $61.0(0)$ & $281.3(0)$ & $39.35(0)$ \\
\hline $217.675(4)$ & $61.0(0)$ & 4.95(8) & $4.47(7)$ \\
\hline $226.023(3)$ & & & $23.9(5)$ \\
\hline $227.858(3)$ & $61.0(0)$ & $17.1(2)$ & $12.4(2)$ \\
\hline $233.635(3)$ & $61.0(0)$ & $8.0(1)$ & $6.84(8)$ \\
\hline $236.033(2)$ & $56.2(6)$ & $112(0)$ & $30.6(3)$ \\
\hline $244.889(4)$ & $61.0(0)$ & $6.86(9)$ & $5.97(8)$ \\
\hline $250.320(3)$ & & & $25.9(4)$ \\
\hline $253.272(5)$ & $61.0(0)$ & $5.23(8)$ & $4.69(7)$ \\
\hline 267.599(4) & $61.0(0)$ & $9.5(1)$ & $7.90(11)$ \\
\hline 269.749(5) & $61.0(0)$ & $9.1(2)$ & $5.70(11)$ \\
\hline $273.270(6)$ & $61.0(0)$ & $1.9(2)$ & $1.65(16)$ \\
\hline $283.822(18)$ & $61.0(0)$ & $0.60(5)$ & $0.59(5)$ \\
\hline
\end{tabular}

TABLE VIII. (Continued.)

\begin{tabular}{|c|c|c|c|}
\hline$E_{r}(\mathrm{eV})$ & $\Gamma_{\gamma}(\mathrm{meV})$ & $g \Gamma_{n}(\mathrm{meV})$ & $\frac{g \Gamma_{\gamma} \Gamma_{n}}{\Gamma}(\mathrm{meV})$ \\
\hline 288.681(3) & $61.0(0)$ & $26.9(5)$ & $17.0(3)$ \\
\hline $296.005(4)$ & $61.0(0)$ & $10.7(2)$ & $8.66(13)$ \\
\hline 297.651(5) & & & $7.19(12)$ \\
\hline $310.672(8)$ & $61.0(0)$ & $6.8(2)$ & $4.70(14)$ \\
\hline $315.757(6)$ & & & $14.7(3)$ \\
\hline $328.800(5)$ & $61.0(0)$ & $16.4(3)$ & $12.1(2)$ \\
\hline $330.393(3)$ & $61.0(0)$ & $8.8(2)$ & $7.38(14)$ \\
\hline $334.430(2)$ & $61.0(0)$ & $11.7(3)$ & $9.32(20)$ \\
\hline $338.904(4)$ & $61.0(0)$ & $39.6(8)$ & $21.2(4)$ \\
\hline $345.618(16)$ & $61.0(0)$ & $3.0(1)$ & $2.84(8)$ \\
\hline $346.891(5)$ & $61.0(0)$ & $18.3(3)$ & $13.1(2)$ \\
\hline $350.903(5)$ & $61.0(0)$ & $32.2(7)$ & $10.4(2)$ \\
\hline $369.115(6)$ & $61.0(0)$ & $25.6(5)$ & $16.4(4)$ \\
\hline $371.890(4)$ & $61.0(0)$ & $13.3(11)$ & $10.3(9)$ \\
\hline $375.223(4)$ & $61.0(0)$ & $6.20(20)$ & 4.41(14) \\
\hline $382.312(6)$ & $61.0(0)$ & $16.1(7)$ & $11.9(6)$ \\
\hline $390.254(6)$ & $61.0(0)$ & $90.0(0)$ & $30.3(0)$ \\
\hline 391.683(9) & $61.0(0)$ & $10.4(2)$ & $8.46(17)$ \\
\hline $398.565(4)$ & $64.1(10)$ & $73.5(0)$ & $29.1(5)$ \\
\hline 403.624(9) & $39.6(15)$ & $99.97(0)$ & $9.01(35)$ \\
\hline $404.880(0)$ & $61.0(0)$ & $12.4(3)$ & $9.75(27)$ \\
\hline $408.307(5)$ & $61.0(0)$ & $300(0)$ & $37.7(0)$ \\
\hline $419.237(13)$ & & & $3.21(10)$ \\
\hline $423.867(44)$ & $61.0(0)$ & $0.72(6)$ & $0.71(6)$ \\
\hline 425.911(4) & $61.0(0)$ & $6.46(44)$ & $5.66(38)$ \\
\hline 431.463(8) & $61.0(0)$ & $38.4(17)$ & $20.9(9)$ \\
\hline $441.858(7)$ & $76.4(13)$ & $79.5(0)$ & $15.4(3)$ \\
\hline $447.480(20)$ & $61.0(0)$ & $1.53(8)$ & $1.48(7)$ \\
\hline $453.322(6)$ & & & $22.4(6)$ \\
\hline $462.381(6)$ & $61.0(0)$ & $34.1(10)$ & $19.6(6)$ \\
\hline 468.304(11) & $61.0(0)$ & $7.76(23)$ & $5.14(15)$ \\
\hline $472.509(10)$ & $61.0(0)$ & $10.3(2)$ & 8.43(17) \\
\hline $483.500(7)$ & $54.6(10)$ & $102(0)$ & $29.2(5)$ \\
\hline 497.703(8) & $61.0(0)$ & $19.5(4)$ & $13.7(3)$ \\
\hline $504.88(1)$ & $61.0(0)$ & $13.5(3)$ & $10.4(2)$ \\
\hline $539.46(1)$ & $61.0(0)$ & $14.3(6)$ & 7.37(29) \\
\hline $542.19(1)$ & $61.0(0)$ & $15.6(4)$ & $11.6(3)$ \\
\hline $543.70(1)$ & $61.0(0)$ & $11.0(3)$ & $8.86(26)$ \\
\hline $547.66(1)$ & $61.0(0)$ & $11.7(3)$ & $9.32(22)$ \\
\hline $548.86(2)$ & $61.0(0)$ & 4.1(2) & $3.26(14)$ \\
\hline $552.40(1)$ & & & $19.4(26)$ \\
\hline $554.15(1)$ & & & $35.4(12)$ \\
\hline $572.28(1)$ & $58.6(11)$ & $64.0(0)$ & $26.1(5)$ \\
\hline $582.41(1)$ & $61.0(0)$ & $15.2(13)$ & $11.4(10)$ \\
\hline $587.04(1)$ & $64.5(15)$ & $72.0(0)$ & $13.2(3)$ \\
\hline $589.00(0)$ & $61.0(0)$ & $2.9(1)$ & 2.74(12) \\
\hline $592.68(1)$ & $56.7(10)$ & $80.5(0)$ & $27.8(5)$ \\
\hline $594.70(0)$ & $61.0(0)$ & $6.0(2)$ & 4.31(16) \\
\hline $598.13(1)$ & $61.0(0)$ & $19.2(5)$ & $13.5(3)$ \\
\hline $599.92(1)$ & $65.0(0)$ & $10.7(2)$ & $8.79(20)$ \\
\hline $606.80(1)$ & $61.0(0)$ & $33.5(9)$ & $19.3(5)$ \\
\hline $608.43(2)$ & $61.0(0)$ & $6.6(4)$ & $4.63(25)$ \\
\hline $610.52(1)$ & & & $26.5(10)$ \\
\hline $622.99(1)$ & $61.0(0)$ & $25.0(6)$ & $16.2(4)$ \\
\hline $625.25(1)$ & $61.0(0)$ & $33.9(10)$ & $19.5(6)$ \\
\hline $628.15(1)$ & & & $22.1(8)$ \\
\hline $636.83(1)$ & $61.0(0)$ & $11.9(3)$ & $9.43(27)$ \\
\hline
\end{tabular}


TABLE VIII. (Continued.)

\begin{tabular}{|c|c|c|c|}
\hline$E_{r}(\mathrm{eV})$ & $\Gamma_{\gamma}(\mathrm{meV})$ & $g \Gamma_{n}(\mathrm{meV})$ & $\frac{g \Gamma_{\gamma} \Gamma_{n}}{\Gamma}(\mathrm{meV})$ \\
\hline $638.29(1)$ & $61.0(0)$ & $29.8(9)$ & $18.0(5)$ \\
\hline $640.12(1)$ & $61.0(13)$ & $81.0(0)$ & $29.3(6)$ \\
\hline $648.63(3)$ & $61.0(0)$ & $3.6(4)$ & $3.36(36)$ \\
\hline $657.20(1)$ & $61.0(0)$ & $23.0(7)$ & $15.3(5)$ \\
\hline $664.79(1)$ & $61.0(0)$ & $18.8(13)$ & $8.43(59)$ \\
\hline $668.87(1)$ & $61.0(0)$ & $7.1(2)$ & $6.15(17)$ \\
\hline $675.05(2)$ & $61.0(0)$ & $3.9(1)$ & $3.61(13)$ \\
\hline $678.43(1)$ & $61.0(0)$ & $31.9(20)$ & $10.3(6)$ \\
\hline $682.12(2)$ & $61.0(0)$ & 7.7(2) & $6.58(18)$ \\
\hline $687.15(1)$ & $61.0(0)$ & $26.9(18)$ & $9.74(64)$ \\
\hline $692.63(4)$ & $61.0(0)$ & 2.7(4) & $2.31(38)$ \\
\hline $693.38(1)$ & $61.0(0)$ & $6.2(11)$ & $5.4(10)$ \\
\hline $698.05(1)$ & $61.0(0)$ & $45.8(16)$ & $22.9(8)$ \\
\hline $702.18(1)$ & $58.9(12)$ & $109.5(0)$ & $31.5(6)$ \\
\hline $710.25(1)$ & $61.0(0)$ & 23.1(6) & $15.4(4)$ \\
\hline $716.05(1)$ & $61.0(0)$ & $24.0(7)$ & $15.7(5)$ \\
\hline $718.00(1)$ & $61.0(0)$ & $27.8(7)$ & $17.3(5)$ \\
\hline $727.87(1)$ & $61.0(0)$ & $31.4(9)$ & $18.6(6)$ \\
\hline $733.60(2)$ & $61.0(0)$ & $15.4(4)$ & $11.5(3)$ \\
\hline $737.19(1)$ & & & $22.7(8)$ \\
\hline $750.12(1)$ & $61.8(17)$ & $75.8(0)$ & $28.8(8)$ \\
\hline $753.72(1)$ & & & $20.2(10)$ \\
\hline $757.43(2)$ & $61.0(0)$ & $30.0(14)$ & $18.1(9)$ \\
\hline $759.00(3)$ & $61.0(0)$ & $15.8(7)$ & $11.7(5)$ \\
\hline $760.79(2)$ & $55.5(15)$ & $285.0(0)$ & $36.3(10)$ \\
\hline $769.28(1)$ & $61.0(0)$ & $14.3(5)$ & $10.9(4)$ \\
\hline $771.18(1)$ & & & $22.7(18)$ \\
\hline $777.48(1)$ & $61.0(0)$ & $34.6(9)$ & $19.7(5)$ \\
\hline $784.46(1)$ & $66.6(16)$ & $85.0(0)$ & $31.5(7)$ \\
\hline 791.74(1) & $73.2(18)$ & $83.0(0)$ & $33.0(8)$ \\
\hline $794.10(2)$ & $61.0(0)$ & 10.0(4) & $8.23(34)$ \\
\hline $799.56(2)$ & $73.6(23)$ & $115.5(0)$ & $15.9(5)$ \\
\hline $801.84(3)$ & $61.0(0)$ & $6.9(3)$ & $5.97(28)$ \\
\hline $804.67(1)$ & & & $37.0(17)$ \\
\hline $810.27(1)$ & $53.7(30)$ & $81.0(0)$ & $26.9(15)$ \\
\hline $826.11(2)$ & $61.0(0)$ & $18.3(5)$ & $13.1(4)$ \\
\hline $831.27(1)$ & & & $24.6(11)$ \\
\hline $838.09(2)$ & $61.0(0)$ & $16.7(6)$ & $12.3(4)$ \\
\hline $840.15(2)$ & $61.0(0)$ & $23.8(10)$ & $15.7(6)$ \\
\hline $842.67(4)$ & $61.0(0)$ & $6.5(7)$ & $5.70(59)$ \\
\hline $847.67(2)$ & $65.7(16)$ & $398(10)$ & $43.9(16)$ \\
\hline $853.76(2)$ & $61.0(0)$ & $3.2(2)$ & $3.0(2)$ \\
\hline $867.96(2)$ & $52.0(18)$ & $80.0(0)$ & $11.2(4)$ \\
\hline $871.68(1)$ & $63.7(16)$ & $80.0(0)$ & $29.9(8)$ \\
\hline $878.45(1)$ & $61.0(0)$ & $27.6(9)$ & $17.2(6)$ \\
\hline $882.89(2)$ & $61.0(0)$ & $10.2(10)$ & $8.3(8)$ \\
\hline $884.58(2)$ & $61.0(0)$ & $12.4(4)$ & $9.78(35)$ \\
\hline $889.53(1)$ & $61.0(0)$ & $27.4(8)$ & $17.1(5)$ \\
\hline $896.36(1)$ & $61.0(0)$ & $12.0(8)$ & $9.48(65)$ \\
\hline $906.01(3)$ & $61.0(0)$ & $9.0(5)$ & $5.65(31)$ \\
\hline $910.61(2)$ & $61.0(0)$ & 14.6(15) & 7.47(76) \\
\hline $916.65(2)$ & $75.6(17)$ & $195.0(0)$ & $43.9(10)$ \\
\hline $919.36(2)$ & $70.6(18)$ & $104.0(0)$ & $35.1(9)$ \\
\hline $929.09(2)$ & & & $24.8(17)$ \\
\hline $929.70(4)$ & $61.0(0)$ & $72.5(0)$ & $12.6(0)$ \\
\hline $940.53(1)$ & $62.3(20)$ & $150.0(0)$ & $35.65(12)$ \\
\hline $942.19(5)$ & $54.7(31)$ & $115.0(0)$ & $12.2(7)$ \\
\hline $947.10(1)$ & $74.3(2.3)$ & $63.5(0)$ & 29.7(9) \\
\hline
\end{tabular}

TABLE VIII. (Continued.)

\begin{tabular}{|c|c|c|c|}
\hline$E_{r}(\mathrm{eV})$ & $\Gamma_{\gamma}(\mathrm{meV})$ & $g \Gamma_{n}(\mathrm{meV})$ & $\frac{g \Gamma_{\gamma} \Gamma_{n}}{\Gamma}(\mathrm{meV})$ \\
\hline $953.37(3)$ & $59.9(2.1)$ & $93.5(0)$ & $12.9(5)$ \\
\hline $957.73(2)$ & $61.0(0)$ & $35.4(18)$ & $20.0(10)$ \\
\hline $962.15(8)$ & $61.0(0)$ & $3.1(3)$ & $2.87(26)$ \\
\hline $964.29(2)$ & & & $28.7(17)$ \\
\hline $975.77(1)$ & $65.0(0)$ & $10.4(13)$ & $8.5(10)$ \\
\hline $981.44(1)$ & $61.0(0)$ & $50.0(0)$ & $23.9(0)$ \\
\hline $986.83(4)$ & $61.0(0)$ & $11.6(8)$ & $6.58(43)$ \\
\hline $989.71(2)$ & $62.7(21)$ & $75.0(0)$ & $28.9(1)$ \\
\hline 1006.01(2) & & & $36.0(24)$ \\
\hline 1016.48(3) & & & $11.4(13)$ \\
\hline $1022.48(2)$ & & & $38.7(22)$ \\
\hline $1030.45(2)$ & & & $16.5(6)$ \\
\hline 1035.96(1) & & & $6.7(12)$ \\
\hline 1039.21(1) & & & $3.0(6)$ \\
\hline 1043.45(4) & & & $9.2(9)$ \\
\hline $1050.62(2)$ & & & $37.4(26)$ \\
\hline 1053.21(2) & & & $22.9(10)$ \\
\hline 1064.60(3) & & & $9.0(6)$ \\
\hline $1069.62(3)$ & & & $9.5(4)$ \\
\hline $1072.37(2)$ & & & $36.9(20)$ \\
\hline $1078.42(4)$ & & & $5.4(3)$ \\
\hline 1089.02(1) & & & $20.7(25)$ \\
\hline 1095.21(1) & & & $27.5(10)$ \\
\hline 1100.02(2) & & & $19.4(8)$ \\
\hline 1104.06(3) & & & $11.0(4)$ \\
\hline 1114.34(2) & & & $39.9(17)$ \\
\hline 1122.43(3) & & & $14.0(7)$ \\
\hline 1135.04(3) & & & $9.1(4)$ \\
\hline 1138.56(2) & & & $31.5(18)$ \\
\hline 1140.77(2) & & & $23.9(21)$ \\
\hline 1148.71(2) & & & $19.4(9)$ \\
\hline $1155.42(5)$ & & & 4.9(3) \\
\hline 1161.15(1) & & & $4.1(8)$ \\
\hline 1164.79(3) & & & $11.1(7)$ \\
\hline 1169.53(2) & & & 20.3(9) \\
\hline 1174.42(4) & & & 7.9(4) \\
\hline 1176.78(2) & & & $7.5(13)$ \\
\hline $1180.20(4)$ & & & $11.7(14)$ \\
\hline $1191.26(2)$ & & & 19.7(9) \\
\hline $1202.02(3)$ & & & $12.9(13)$ \\
\hline 1209.69(4) & & & $41.3(25)$ \\
\hline $1210.73(8)$ & & & $12.0(22)$ \\
\hline $1213.08(11)$ & & & $3.1(3)$ \\
\hline $1223.34(2)$ & & & $29.3(23)$ \\
\hline 1226.01(3) & & & $19.6(10)$ \\
\hline $1228.94(4)$ & & & $7.7(6)$ \\
\hline 1239.43(4) & & & $43.8(0)$ \\
\hline $1240.05(8)$ & & & $37.6(46)$ \\
\hline $1241.05(5)$ & & & $32.3(55)$ \\
\hline 1244.72(3) & & & $3.7(7)$ \\
\hline 1255.70(2) & & & $41.1(23)$ \\
\hline $1265.14(6)$ & & & $6.3(4)$ \\
\hline $1268.19(4)$ & & & $17.6(9)$ \\
\hline $1270.00(5)$ & & & $10.9(7)$ \\
\hline $1289.20(2)$ & & & $33.2(35)$ \\
\hline 1293.86(3) & & & $34.1(41)$ \\
\hline 1296.98(3) & & & $23.5(14)$ \\
\hline 1301.19(3) & & & $41.0(26)$ \\
\hline
\end{tabular}


TABLE VIII. (Continued.)

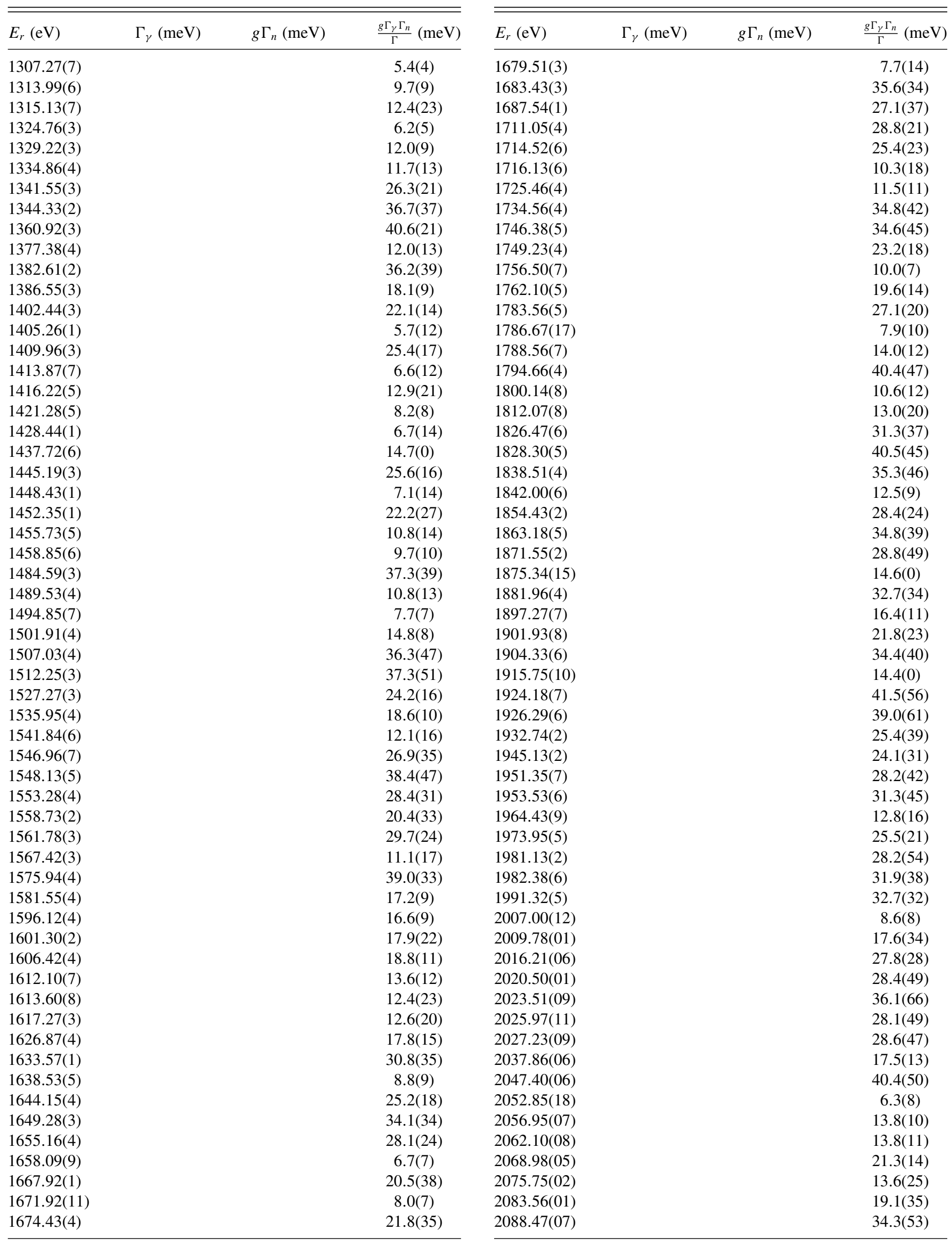

TABLE VIII. (Continued.) 
TABLE VIII. (Continued.)

\begin{tabular}{|c|c|c|}
\hline$E_{r}(\mathrm{eV})$ & $\Gamma_{\gamma}(\mathrm{meV}) \quad g \Gamma_{n}(\mathrm{meV})$ & $\frac{g \Gamma_{\gamma} \Gamma_{n}}{\Gamma}(\mathrm{meV})$ \\
\hline 2091.77(07) & & $35.6(43)$ \\
\hline $2094.39(12)$ & & $13.4(16)$ \\
\hline 2099.82(05) & & $39.1(48)$ \\
\hline $2105.19(03)$ & & $9.6(18)$ \\
\hline $2115.83(07)$ & & $19.5(35)$ \\
\hline $2120.12(06)$ & & $19.3(14)$ \\
\hline $2128.20(03)$ & & $9.3(18)$ \\
\hline $2133.18(11)$ & & $10.6(11)$ \\
\hline $2137.15(06)$ & & $26.6(21)$ \\
\hline $2140.43(13)$ & & $7.2(8)$ \\
\hline $2153.12(18)$ & & $9.6(15)$ \\
\hline 2155.44(08) & & $41.4(53)$ \\
\hline $2161.22(02)$ & & $8.7(17)$ \\
\hline $2166.75(01)$ & & $6.6(13)$ \\
\hline $2169.68(01)$ & & $16.3(31)$ \\
\hline $2187.80(06)$ & & $41.3(58)$ \\
\hline 2192.23(04) & & $18.2(34)$ \\
\hline 2195.66(06) & & $37.7(67)$ \\
\hline 2201.91(06) & & $10.2(17)$ \\
\hline $2207.86(07)$ & & $39.1(62)$ \\
\hline $2211.57(08)$ & & $37.9(60)$ \\
\hline $2214.81(14)$ & & $11.9(11)$ \\
\hline $2219.74(01)$ & & $11.4(22)$ \\
\hline $2226.59(02)$ & & $25.0(44)$ \\
\hline $2234.70(07)$ & & $42.2(51)$ \\
\hline $2239.59(08)$ & & $21.9(22)$ \\
\hline $2243.00(00)$ & & $14.1(11)$ \\
\hline $2256.28(06)$ & & $42.8(29)$ \\
\hline 2269.81(14) & & $10.4(10)$ \\
\hline $2273.75(08)$ & & $24.8(22)$ \\
\hline $2280.67(07)$ & & $34.9(53)$ \\
\hline $2284.09(07)$ & & $32.2(40)$ \\
\hline $2291.08(01)$ & & $21.9(41)$ \\
\hline $2293.36(08)$ & & $33.7(52)$ \\
\hline 2299.87(08) & & $39.5(59)$ \\
\hline $2304.73(10)$ & & $41.5(55)$ \\
\hline 2306.89(09) & & $41.5(50)$ \\
\hline $2330.18(03)$ & & $38.4(45)$ \\
\hline $2343.50(00)$ & & $18.8(14)$ \\
\hline $2351.55(02)$ & & $36.3(36)$ \\
\hline $2360.96(09)$ & & $16.6(14)$ \\
\hline $2367.67(01)$ & & $34.2(58)$ \\
\hline 2374.30(08) & & $27.2(30)$ \\
\hline $2377.58(01)$ & & $13.9(27)$ \\
\hline $2386.96(01)$ & & $6.4(13)$ \\
\hline $2393.40(10)$ & & $24.5(26)$ \\
\hline $2400.21(07)$ & & $37.6(56)$ \\
\hline $2405.97(17)$ & & $8.1(9)$ \\
\hline $2411.79(07)$ & & $38.8(47)$ \\
\hline $2424.85(02)$ & & $28.0(52)$ \\
\hline 2431.75(09) & & $39.2(72)$ \\
\hline $2434.28(01)$ & & $15.4(30)$ \\
\hline $2438.55(01)$ & & $6.5(13)$ \\
\hline $2443.00(11)$ & & $38.2(73)$ \\
\hline $2444.60(11)$ & & $41.8(70)$ \\
\hline 2448.73(02) & & $11.9(23)$ \\
\hline $2461.62(07)$ & & $30.8(37)$ \\
\hline $2467.17(02)$ & & $11.5(22)$ \\
\hline
\end{tabular}

TABLE VIII. (Continued.)

\begin{tabular}{|c|c|c|}
\hline$E_{r}(\mathrm{eV})$ & $g \Gamma_{n}(\mathrm{meV})$ & $\frac{g \Gamma_{\gamma} \Gamma_{n}}{\Gamma}(\mathrm{meV})$ \\
\hline 2471.07(09) & & $39.8(71)$ \\
\hline $2471.46(08)$ & & $39.8(69)$ \\
\hline 2482.92(03) & & $35.6(67)$ \\
\hline 2485.02(09) & & $38.8(68)$ \\
\hline 2500.94(10) & & $16.9(15)$ \\
\hline $2512.76(08)$ & & $39.5(55)$ \\
\hline 2518.62(07) & & $30.5(44)$ \\
\hline $2520.60(21)$ & & $30.0(50)$ \\
\hline 2524.07(10) & & $22.3(22)$ \\
\hline 2536.81(08) & & $38.5(48)$ \\
\hline $2540.18(01)$ & & $17.0(33)$ \\
\hline $2545.36(14)$ & & $15.9(16)$ \\
\hline $2553.85(08)$ & & $27.5(40)$ \\
\hline $2555.49(05)$ & & $29.3(53)$ \\
\hline $2563.41(11)$ & & $29.7(55)$ \\
\hline $2564.88(07)$ & & $26.7(36)$ \\
\hline $2571.29(02)$ & & 7.6(15) \\
\hline $2576.65(13)$ & & $26.9(49)$ \\
\hline 2578.13(03) & & $30.7(53)$ \\
\hline $2583.14(02)$ & & $11.2(22)$ \\
\hline $2588.15(07)$ & & $33.3(49)$ \\
\hline $2600.98(07)$ & & $37.4(52)$ \\
\hline 2610.11(07) & & $42.2(49)$ \\
\hline $2615.00(00)$ & & $12.3(14)$ \\
\hline $2620.38(08)$ & & $42.8(48)$ \\
\hline 2627.62(02) & & $13.4(27)$ \\
\hline 2630.20(10) & & $40.3(60)$ \\
\hline $2637.19(01)$ & & $11.4(23)$ \\
\hline $2641.14(08)$ & & $38.2(52)$ \\
\hline $2661.48(02)$ & & $26.7(50)$ \\
\hline 2666.79(02) & & $17.5(36)$ \\
\hline 2671.04(12) & & $16.1(15)$ \\
\hline 2684.53(11) & & $37.9(65)$ \\
\hline $2686.53(11)$ & & $40.9(69)$ \\
\hline 2695.89(02) & & $29.3(54)$ \\
\hline 2701.72(02) & & 17.1(33) \\
\hline 2711.62(11) & & $39.0(79)$ \\
\hline 2713.96(09) & & $36.8(74)$ \\
\hline $2720.15(09)$ & & $28.4(33)$ \\
\hline 2730.91(10) & & $21.5(20)$ \\
\hline $2742.43(10)$ & & $37.9(49)$ \\
\hline $2746.50(10)$ & & $35.7(60)$ \\
\hline $2751.96(08)$ & & $35.5(57)$ \\
\hline $2756.14(21)$ & & $11.7(16)$ \\
\hline $2765.30(08)$ & & $38.2(67)$ \\
\hline 2774.91(10) & & $38.4(70)$ \\
\hline 2778.04(16) & & $17.9(21)$ \\
\hline 2785.21(10) & & $35.0(52)$ \\
\hline 2790.70(10) & & $38.7(54)$ \\
\hline $2801.00(07)$ & & $38.5(53)$ \\
\hline 2810.27(10) & & $41.4(52)$ \\
\hline 2816.92(14) & & $20.9(24)$ \\
\hline 2821.74(18) & & $27.9(43)$ \\
\hline 2823.03(12) & & $28.2(43)$ \\
\hline $2832.47(11)$ & & $20.8(20)$ \\
\hline 2839.82(01) & & 7.0(14) \\
\hline $2848.55(01)$ & & $13.3(27)$ \\
\hline $2857.43(02)$ & & $29.8(51)$ \\
\hline
\end{tabular}


TABLE VIII. (Continued.)

\begin{tabular}{|c|c|c|}
\hline$E_{r}(\mathrm{eV})$ & $g \Gamma_{n}(\mathrm{meV})$ & $\frac{g \Gamma_{\gamma} \Gamma_{n}}{\Gamma}(\mathrm{meV})$ \\
\hline $2868.97(01)$ & & $29.4(52)$ \\
\hline 2877.51(13) & & $38.6(60)$ \\
\hline $2882.00(10)$ & & $39.8(61)$ \\
\hline $2888.79(10)$ & & $30.6(41)$ \\
\hline 2902.61(12) & & $39.9(75)$ \\
\hline 2907.21(11) & & $39.6(73)$ \\
\hline $2913.23(01)$ & & $9.0(18)$ \\
\hline 2926.03(16) & & $14.6(16)$ \\
\hline $2930.70(02)$ & & $11.3(22)$ \\
\hline $2939.07(15)$ & & $28.2(40)$ \\
\hline $2940.88(02)$ & & $27.4(51)$ \\
\hline $2952.73(05)$ & & $35.2(64)$ \\
\hline $2956.29(12)$ & & $37.5(71)$ \\
\hline 2968.61(08) & & $37.6(54)$ \\
\hline 2980.97(11) & & $37.7(71)$ \\
\hline 2985.92(13) & & $36.5(70)$ \\
\hline 2991.48(16) & & $40.0(81)$ \\
\hline $2993.54(14)$ & & $41.3(76)$ \\
\hline
\end{tabular}

All spins are defined as $s$ wave. Parameters with zero uncertainty were treated as fixed values in the SAMMY fits.

TABLE IX. Resonance parameters of ${ }^{188}$ Os.

\begin{tabular}{|c|c|c|c|}
\hline$E_{r}(\mathrm{eV})$ & $\Gamma_{\gamma}(\mathrm{meV})$ & $g \Gamma_{n}(\mathrm{meV})$ & $\frac{g \Gamma_{\gamma} \Gamma_{n}}{\Gamma}(\mathrm{meV})$ \\
\hline $38.68(0)$ & & & $22.1(0)$ \\
\hline $78.739(2)$ & $35.6(5)$ & $377.9(0)$ & $32.5(4)$ \\
\hline $150.066(3)$ & $37.4(2)$ & $159.8(10)$ & $30.3(3)$ \\
\hline 191.922(3) & $36.1(3)$ & $120.0(10)$ & $27.7(3)$ \\
\hline $254.208(3)$ & & & $31.1(4)$ \\
\hline 282.441(3) & $52.0(0)$ & 12.3(1) & $9.95(12)$ \\
\hline $317.23(0)$ & $57.5(5)$ & $960.8(0)$ & $54.3(4)$ \\
\hline $388.37(2)$ & $43.8(5)$ & $812.2(0)$ & $41.5(5)$ \\
\hline $478.92(2)$ & $43.5(5)$ & 490.1(0) & $40.0(5)$ \\
\hline $528.774(5)$ & & & $37.7(8)$ \\
\hline $536.97(1)$ & & & $36.8(8)$ \\
\hline $620.55(1)$ & & & $40.6(9)$ \\
\hline $649.55(1)$ & & & $37.1(12)$ \\
\hline $705.99(1)$ & & & $38.0(13)$ \\
\hline $745.70(2)$ & $48.6(7)$ & 313(0) & $42.1(6)$ \\
\hline $781.51(5)$ & $44.6(10)$ & $1125(0)$ & $42.9(9)$ \\
\hline $819.95(1)$ & & & $28.7(15)$ \\
\hline $844.41(6)$ & $44.6(10)$ & 1870.(0) & $43.5(9)$ \\
\hline $863.11(1)$ & $44.0(8)$ & $380(0)$ & $39.5(8)$ \\
\hline $900.27(2)$ & $52.0(0)$ & $2.9(5)$ & $2.79(51)$ \\
\hline $980.03(1)$ & $46.5(9)$ & $140.0(0)$ & $34.9(7)$ \\
\hline $1001.523(3)$ & $52.0(0)$ & $41.0(0)$ & $22.9(0)$ \\
\hline $1042.15(2)$ & $48.0(7)$ & $529(0)$ & $44.0(7)$ \\
\hline 1079.76(1) & $52.0(0)$ & $20.2(5)$ & $14.5(4)$ \\
\hline $1107.78(4)$ & $51.9(8)$ & 1280.(0) & $49.9(8)$ \\
\hline 1182.74(1) & $52.0(0)$ & $43.0(0)$ & $23.5(0)$ \\
\hline 1216.67(1) & & & $47.5(30)$ \\
\hline 1293.22(2) & $52.0(0)$ & $86.0(0)$ & $32.4(0)$ \\
\hline 1316.53(3) & $52.1(10)$ & $936(0)$ & $49.3(9)$ \\
\hline 1347.89(2) & $54.1(18)$ & $170.0(0)$ & $41.0(14)$ \\
\hline $1413.27(5)$ & $59.5(12)$ & $1330(0)$ & $57.0(11)$ \\
\hline
\end{tabular}

TABLE IX. (Continued.)

\begin{tabular}{|c|c|c|c|}
\hline$E_{r}(\mathrm{eV})$ & $\Gamma_{\gamma}(\mathrm{meV})$ & $g \Gamma_{n}(\mathrm{meV})$ & $\frac{g \Gamma_{\gamma} \Gamma_{n}}{\Gamma}(\mathrm{meV})$ \\
\hline $1485.60(2)$ & & & $37.9(58)$ \\
\hline $1520.35(2)$ & $48.1(13)$ & $122.0(0)$ & $34.5(10)$ \\
\hline $1544.931(4)$ & $52.0(0)$ & $52.0(0)$ & $26.0(0)$ \\
\hline $1598.14(7)$ & $51.4(11)$ & $1730(0)$ & $49.9(11)$ \\
\hline $1625.26(1)$ & $52.0(0)$ & $3.1(6)$ & $2.92(57)$ \\
\hline 1673.73(4) & $47.1(10)$ & $678.1(0)$ & 44.1(9) \\
\hline $1719.94(2)$ & $54.3(14)$ & $113.0(0)$ & $36.7(10)$ \\
\hline 1764.43(1) & $52.0(0)$ & $5.0(10)$ & $4.59(90)$ \\
\hline $1779.50(3)$ & $52.0(0)$ & $63.0(0)$ & $28.49(0)$ \\
\hline 1803.43(2) & $57.8(14)$ & $202.0(0)$ & $44.9(11)$ \\
\hline $1876.87(6)$ & $45.2(11)$ & $1020(0)$ & $43.3(11)$ \\
\hline $1905.748(3)$ & $52.0(0)$ & $42.0(0)$ & $23.2(0)$ \\
\hline $1967.62(1)$ & $52.0(0)$ & $113.0(0)$ & $35.6(0)$ \\
\hline 1971.65(3) & $47.3(13)$ & $303.9(0)$ & $41.0(11)$ \\
\hline 2017.89(3) & & & $32.2(24)$ \\
\hline 2049.07(9) & $52.0(0)$ & $5.9(4)$ & $5.33(36)$ \\
\hline 2092.24(3) & $55.0(15)$ & $219.0(0)$ & $44.0(12)$ \\
\hline 2138.05(3) & $56.3(16)$ & $306.0(0)$ & 47.6(14) \\
\hline $2179.42(2)$ & $52.0(0)$ & 21.4(38) & $15.2(27)$ \\
\hline 2191.59(5) & $50.6(17)$ & $351.1(0)$ & $44.2(15)$ \\
\hline 2257.79(1) & $52.0(0)$ & $5.0(10)$ & $4.57(91)$ \\
\hline $2274.38(5)$ & $48.0(13)$ & $540.9(0)$ & $44.1(12)$ \\
\hline 2298.98(8) & $50.1(15)$ & 1360.(0) & $48.3(14)$ \\
\hline 2386.82(1) & $52.0(0)$ & $93(17)$ & $33.4(61)$ \\
\hline 2412.06(7) & $47.1(15)$ & $762(0)$ & $44.4(14)$ \\
\hline 2438.33(4) & $52.6(17)$ & $233(0)$ & $42.9(14)$ \\
\hline $2500.93(7)$ & $45.3(14)$ & $715(0)$ & $42.6(13)$ \\
\hline 2504.78(3) & $52.0(0)$ & $2.8(6)$ & $2.70(54)$ \\
\hline $2545.05(1)$ & $52.0(0)$ & $53(10)$ & $26.2(50)$ \\
\hline $2570.83(2)$ & $46.8(16)$ & 267.0(0) & $39.8(13)$ \\
\hline 2613.22(15) & $52.0(0)$ & $5.7(6)$ & $5.10(55)$ \\
\hline 2619.76(1) & $52.0(0)$ & $4.0(8)$ & $3.71(74)$ \\
\hline $2626.82(4)$ & $55.5(22)$ & $253.0(0)$ & $45.5(18)$ \\
\hline $2729.12(12)$ & $52.0(0)$ & $9.6(7)$ & $8.07(59)$ \\
\hline $2768.44(1)$ & $52.0(0)$ & $30(6)$ & $19.0(38)$ \\
\hline 2799.50(11) & $52.0(0)$ & $11.6(8)$ & $9.52(7)$ \\
\hline $2815.79(6)$ & & & $41(6)$ \\
\hline 2864.00(6) & & & 36.1(38) \\
\hline 2924.14(2) & $52.0(0)$ & $10(2)$ & 8.4(17) \\
\hline 2967.98(8) & $52.0(0)$ & $5.2(10)$ & 4.73(87) \\
\hline $2975.72(13)$ & $47.3(19)$ & $1670(0)$ & $46.0(18)$ \\
\hline $2988.42(6)$ & & & $38.9(48)$ \\
\hline $3036.60(7)$ & $58.4(18)$ & $600(0)$ & $53.2(17)$ \\
\hline 3056.03(8) & $62.7(20)$ & $730(0)$ & 57.7(19) \\
\hline $3113.30(8)$ & $54.8(20)$ & 403(0) & $48.3(17)$ \\
\hline $3128.95(8)$ & $58.3(22)$ & $210(0)$ & $45.6(18)$ \\
\hline $3186.54(12)$ & $55.7(24)$ & $4390(0)$ & $55.0(24)$ \\
\hline $3208.81(8)$ & $55.9(22)$ & 437(0) & 49.6(19) \\
\hline 3269.43(9) & & & $25.2(22)$ \\
\hline $3284.27(7)$ & $51.4(18)$ & $448(0)$ & $46.1(16)$ \\
\hline 3355.03(6) & $61.1(24)$ & $237(0)$ & $48.6(19)$ \\
\hline $3417.95(8)$ & $56.9(21)$ & $360(0)$ & 49.1(19) \\
\hline $3438.86(8)$ & $49.8(19)$ & $528(0)$ & $45.5(17)$ \\
\hline $3486.12(8)$ & & & $27.8(23)$ \\
\hline $3517.56(01)$ & $52.0(0)$ & $7.0(14)$ & $6.2(12)$ \\
\hline $3600.22(10)$ & $59.7(22)$ & $786(0)$ & $55.5(21)$ \\
\hline $3632.02(01)$ & $52.0(0)$ & $10(2)$ & $8.4(17)$ \\
\hline $3660.31(01)$ & $52.0(0)$ & $15(3)$ & $11.7(23)$ \\
\hline
\end{tabular}


TABLE IX. (Continued.)

\begin{tabular}{|c|c|c|c|}
\hline$E_{r}(\mathrm{eV})$ & $\Gamma_{\gamma}(\mathrm{meV})$ & $g \Gamma_{n}(\mathrm{meV})$ & $\frac{g \Gamma_{\gamma} \Gamma_{n}}{\Gamma}(\mathrm{meV})$ \\
\hline $3668.31(02)$ & $52.0(0)$ & $20.2(39)$ & $14.5(28)$ \\
\hline $3695.02(20)$ & $44.9(24)$ & $1790(0)$ & $43.8(24)$ \\
\hline $3706.35(52)$ & $35.2(3)$ & $4590(0)$ & $34.9(30)$ \\
\hline $3719.78(11)$ & $55.9(25)$ & $763(0)$ & $52.0(23)$ \\
\hline $3732.94(02)$ & $52.0(0)$ & 9.3(18) & 7.9(15) \\
\hline $3772.65(13)$ & & & $22.9(22)$ \\
\hline $3883.98(10)$ & $62.1(24)$ & $703(0)$ & $57.1(22)$ \\
\hline $3929.04(01)$ & $52.0(0)$ & $20.3(40)$ & $14.6(29)$ \\
\hline $3931.48(01)$ & $52.0(0)$ & $92.0(0)$ & $33.2(0)$ \\
\hline $3947.53(10)$ & $56.3(31)$ & $134.0(0)$ & $39.6(22)$ \\
\hline $3966.66(10)$ & $59.0(36)$ & $111.0(0)$ & $38.5(24)$ \\
\hline $3982.07(03)$ & $52.0(0)$ & $19.8(40)$ & $14.5(29)$ \\
\hline $3987.38(12)$ & $52.0(0)$ & $109.0(0)$ & $35.2(0)$ \\
\hline $4106.33(12)$ & $52.0(0)$ & $196(35)$ & 41.1(74) \\
\hline $4134.73(01)$ & & & $34.4(58)$ \\
\hline $4215.40(10)$ & $65.1(33)$ & $208(0)$ & $49.6(25)$ \\
\hline $4236.28(17)$ & $55.9(24)$ & $1451(0)$ & $53.8(24)$ \\
\hline 4268.21(19) & $46.7(24)$ & $1600(0)$ & $45.4(23)$ \\
\hline $4313.22(12)$ & & & $33.8(40)$ \\
\hline $4341.27(14)$ & & & $24.4(23)$ \\
\hline $4434.27(06)$ & $58(10)$ & $263.0(0)$ & $47.8(77)$ \\
\hline $4450.93(16)$ & $55.9(26)$ & $1310(0)$ & $53.7(25)$ \\
\hline $4483.01(16)$ & $52.0(0)$ & $65.0(0)$ & $28.9(0)$ \\
\hline $4579.92(12)$ & $61(6)$ & $130(23)$ & $41.3(85)$ \\
\hline $4603.79(06)$ & & & $25.2(48)$ \\
\hline $4628.01(02)$ & $52.0(0)$ & $10(2)$ & $8.4(17)$ \\
\hline $4641.47(25)$ & $50.5(29)$ & $2150(0)$ & $49.4(28)$ \\
\hline $4722.68(15)$ & $50.7(30)$ & 198(0) & $40.4(24)$ \\
\hline $4746.96(14)$ & $56.0(33)$ & $242(0)$ & $45.5(26)$ \\
\hline $4817.55(11)$ & $54.2(83)$ & $160(0)$ & $40.5(62)$ \\
\hline $4852.49(20)$ & $50.0(30)$ & $300(0)$ & $42.9(26)$ \\
\hline $4881.95(20)$ & $50.2(29)$ & $950(0)$ & $47.7(27)$ \\
\hline $4893.85(15)$ & $56.8(35)$ & $269(0)$ & $46.9(29)$ \\
\hline $4933.39(16)$ & & & $32.4(60)$ \\
\hline $4959.80(08)$ & $47.9(77)$ & 194(0) & $38.4(62)$ \\
\hline $4989.75(08)$ & & & $36.8(60)$ \\
\hline $5054.83(17)$ & & & $44.0(80)$ \\
\hline $5094.82(04)$ & & & $11.8(23)$ \\
\hline $5102.32(07)$ & & & 47.7(69) \\
\hline $5163.11(17)$ & & & $50.3(0)$ \\
\hline $5204.25(03)$ & & & $22.8(45)$ \\
\hline $5211.34(02)$ & & & $28.8(55)$ \\
\hline $5246.54(20)$ & & & $41.3(70)$ \\
\hline $5273.56(03)$ & & & $37.0(71)$ \\
\hline $5337.24(02)$ & & & $13.3(27)$ \\
\hline $5350.27(02)$ & & & $40.7(77)$ \\
\hline $5383.97(01)$ & & & $25.2(51)$ \\
\hline $5401.93(18)$ & & & $45.5(89)$ \\
\hline $5437.43(19)$ & & & $47.4(92)$ \\
\hline $5455.92(19)$ & & & $38.3(60)$ \\
\hline $5525.35(25)$ & & & $47.6(90)$ \\
\hline $5562.64(26)$ & & & $50.1(70)$ \\
\hline $5613.52(03)$ & & & $38.4(71)$ \\
\hline $5634.56(01)$ & & & $25.9(51)$ \\
\hline $5666.13(19)$ & & & $42.3(71)$ \\
\hline $5734.31(23)$ & & & $47.8(74)$ \\
\hline $5785.24(02)$ & & & $44.6(76)$ \\
\hline
\end{tabular}

TABLE IX. (Continued.)

\begin{tabular}{|c|c|c|}
\hline$E_{r}(\mathrm{eV})$ & $g \Gamma_{n}(\mathrm{meV})$ & $\frac{g \Gamma_{\gamma} \Gamma_{n}}{\Gamma}(\mathrm{meV})$ \\
\hline $5829.58(25)$ & & $46.9(89)$ \\
\hline $5890.21(23)$ & & $41.9(80)$ \\
\hline $5907.67(27)$ & & $50.3(0)$ \\
\hline $5971.09(28)$ & & 47.1(93) \\
\hline $5996.10(31)$ & & $46.8(86)$ \\
\hline 6044.67(11) & & $42.1(80)$ \\
\hline $6104.952(4)$ & & $34.4(68)$ \\
\hline $6130.69(01)$ & & $14.4(29)$ \\
\hline $6183.73(28)$ & & $46.4(89)$ \\
\hline $6200.98(42)$ & & $51.0(76)$ \\
\hline $6250.33(29)$ & & $48.2(91)$ \\
\hline $6287.38(25)$ & & $24.5(39)$ \\
\hline $6335.08(36)$ & & $46.5(83)$ \\
\hline $6376.45(24)$ & & $41.8(74)$ \\
\hline $6426.43(22)$ & & $46.6(88)$ \\
\hline $6458.35(04)$ & & $31.6(63)$ \\
\hline $6485.52(25)$ & & $43.4(81)$ \\
\hline $6531.91(46)$ & & $45.8(90)$ \\
\hline $6536.70(29)$ & & $37.3(69)$ \\
\hline $6552.94(38)$ & & $11.6(20)$ \\
\hline $6605.59(10)$ & & $39.1(71)$ \\
\hline $6640.15(01)$ & & $37.9(73)$ \\
\hline 6689.60(12) & & $34.0(66)$ \\
\hline 6711.04(31) & & $46.8(90)$ \\
\hline $6776.33(02)$ & & $38.9(76)$ \\
\hline $6815.15(30)$ & & $43.8(81)$ \\
\hline 6899.58(04) & & $44.9(87)$ \\
\hline 6943.11(02) & & $43.1(84)$ \\
\hline $6959.13(29)$ & & $43.1(77)$ \\
\hline 6993.97(01) & & $20.9(42)$ \\
\hline $7021.82(30)$ & & $46.2(88)$ \\
\hline 7067.64(01) & & $34.5(68)$ \\
\hline $7123.46(05)$ & & $44.9(87)$ \\
\hline $7161.65(01)$ & & $30.1(60)$ \\
\hline 7193.70(03) & & $42.5(77)$ \\
\hline $7233.28(34)$ & & $43.3(82)$ \\
\hline $7260.27(34)$ & & $46.7(92)$ \\
\hline $7279.47(28)$ & & $44.6(83)$ \\
\hline $7325.67(34)$ & & $45.4(88)$ \\
\hline $7372.49(05)$ & & $32.8(66)$ \\
\hline $7384.21(02)$ & & $43.3(83)$ \\
\hline $7443.22(36)$ & & $42.4(77)$ \\
\hline $7475.32(03)$ & & $41.5(81)$ \\
\hline $7501.36(08)$ & & $34.5(68)$ \\
\hline $7546.29(01)$ & & $30.0(60)$ \\
\hline $7585.96(02)$ & & $39.0(76)$ \\
\hline $7641.17(41)$ & & $42.4(73)$ \\
\hline $7700.73(03)$ & & $30.0(60)$ \\
\hline $7733.53(38)$ & & $41.9(75)$ \\
\hline $7779.48(03)$ & & $40.8(79)$ \\
\hline $7877.25(49)$ & & $38.3(65)$ \\
\hline 7891.27(01) & & $27.9(56)$ \\
\hline $7960.26(42)$ & & $34.5(47)$ \\
\hline
\end{tabular}

All spins are defined as $s$ wave. Parameters with zero uncertainty were treated as fixed values in the SAMMY fits. 
[1] M. Galeazzi, F. Fontanelli, F. Gatti, and S. Vitale, Phys. Rev. C 63, 014302 (2000).

[2] R. Gallino, C. Arlandini, M. Busso, M. Lugaro, C. Travaglio, O. Straniero, O. A. Chieffi, and M. Limongi, Astrophys. J. 497, 388 (1998)

[3] C. Arlandini, F. Käppeler, K. Wisshak, R. Gallino, M. Lugaro, M. Busso, and O. Straniero, Astrophys. J. 525, 886 (1999).

[4] C. Sneden, A. McWilliam, G. W. Preston, J. J. Cowan, D. L. Burris, and B. J. Armosky, Astrophys. J. 467, 819 (1996).

[5] C. Sneden, J. Cowan, D. Burris, and J. Truran, Astrophys. J. 496, 235 (1998).

[6] C. Sneden et al., Astrophys. J. 591, 936 (2003).

[7] D. D. Clayton, Astrophys. J. 139, 637 (1964).

[8] J.-M. Luck, J.-L. Birck, and C.-J. Allegre, Nature 283, 256 (1980).

[9] J.-M. Luck, J.-L. Birck, and C.-J. Allegre, Nature 302, 130 (1983).

[10] K. Takahashi, Nucl. Phys. A 718, 325c (2003).

[11] K. Takahashi, in The Future Astronuclear Physics, edited by A. Jorissen et al. (EDP Sciences, Les Ulis, France, 2004), Vol. 11, p. 199.

[12] M. Arnould and K. Takahashi, Rep. Prog. Phys. 62, 395 (1999).

[13] K. Yokoi, K. Takahashi, and M. Arnould, Astron. Astrophys. 117, 65 (1983).

[14] K. Takahashi and K. Yokoi, Nucl. Phys. A 404, 578 (1983).

[15] F. Bosch et al., Phys. Rev. Lett. 77, 5190 (1996).

[16] T. Faestermann, T. Takahashi, P. Kienle, and F. Bosch, in Nuclear Astrophysics, edited by M. Buballa et al. (GSI, Darmstadt, 1998), p. 417.

[17] K. Takahashi, in Tours Symposium on Nuclear Physics III, edited by M. Arnould et al. (AIP, New York, 1998), p. 616.

[18] M. Mosconi et al., Phys. Rev. C 82, 015802 (2010).

[19] M. Mosconi, M. Heil, F. Käppeler, and A. Mengoni, Phys. Rev. C 82, 015803 (2010).

[20] W. Hauser and H. Feshbach, Phys. Rev. 87, 366 (1952).

[21] U. Abbondanno et al., Technical Report CERN-SL-2002-053 ECT, CERN, Geneva, Switzerland.

[22] U. Abbondanno et al., Nucl. Instrum. Methods A 521, 454 (2004).

[23] N. Larson, Technical Report No. ORNL/TM-9179/R6, Oak Ridge National Laboratory (unpublished).

[24] K. Fujii, Ph.D. thesis, University of Padua, 2009.
[25] S. F. Mughabghab, Atlas of Neutron Resonances, 5th ed. (Elsevier, Amsterdam, 2006).

[26] S. Kopecky, P. Siegler, and A. Moens, in Nuclear Data for Science and Technology, edited by O. Bersillon et al. (EDP Sciences, Paris, 2008), p. 623.

[27] S. Kopecky (private communication).

[28] M. S. Moore, J. D. Moses, G. A. Keyworth, J. W. T. Dabbs, and N. W. Hill, Phys. Rev. C 18, 1328 (1978).

[29] J. A. Holmes, S. E. Woosley, W. A. Fowler, and B. A. Zimmerman, At. Data Nucl. Data Tables 18, 305 (1976).

[30] M. Harris, Astrophys. Space Sci. 77, 357 (1981).

[31] T. Rauscher and F.-K. Thielemann, At. Data Nucl. Data Tables 79, 47 (2001).

[32] M. Arnould and S. Goriely, Nucl. Phys. A 777, 157 (2006).

[33] A. Koning, S. Hilaire, and M. Duijvestijn, in International Conference on Nuclear Data for Science and Technology, AIP Conference Series 769, edited by R. Haight et al. (American Institute of Physics, New York, 2005), p. 1154.

[34] T. Belgya et al., Technical Report, International Atomic Energy Agency, Vienna, Austria [http://www-nds.iaea.org/RIPL-2/].

[35] P. Moldauer, Phys. Rev. C 11, 426 (1975).

[36] A. Gilbert and A. G. W. Cameron, Can. J. Phys. 43, 1446 (1965).

[37] B. L. Berman, D. D. Faul, R. A. Alvarez, P. Meyer, and D. L. Olson, Phys. Rev. C 19, 1205 (1979).

[38] ENSDF Compilation, International Atomic Energy Agency, Vienna, Austria [http://www-nds.iaea.or.at/ensdf/].

[39] R. R. Winters, R. L. Macklin, and J. Halperin, Phys. Rev. C 21, 563 (1980).

[40] J. C. Browne and B. L. Berman, Phys. Rev. C 23, 1434 (1981).

[41] M. Segawa, T. Masaki, Y. Nagai, Y. Temma, T. Shima, K. Mishima, M. Igashira, S. Goriely, A. Koning, and S. Hilaire, Phys. Rev. C 76, 022802(R) (2007).

[42] W. A. Fowler and F. Hoyle, Ann. Phys. 10, 280 (1960).

[43] M. Mosconi et al., J. Phys. G: Nucl. Part. Phys. 35, 014015 (2008).

[44] F. De Bernardis, A. Melchiorri, L. Verde, and R. Jimenez, J. Cosmol. Astropart. Phys. 03 (2008) 020.

[45] W. L. Freedman et al., Astrophys. J. 553, 47 (2001).

[46] L. M. Krauss and B. Chaboyer, Science 299, 65 (2003).

[47] C. L. Bennett et al., Astrophys. J. Suppl. 148, 1 (2003). 